\title{
Instabilities of vibroequilibria in rectangular containers
}

\author{
J. Fernández, I. Tinao, J. Porter, and A. Laverón-Simavilla \\ Escuela Técnica Superior de Ingeniería Aeronáutica y del Espacio, Universidad Politécnica de Madrid, \\ 28040 Madrid, Spain
}

(Received 6 October 2016; accepted 3 February 2017; published online 27 February 2017)

\begin{abstract}
Vibroequilibria theory, based on minimizing an averaged energy functional, predicts the quasiequilibrium shape that a fluid volume will take when subjected to high-frequency vibrations. Here we present a detailed comparison of the predictions of vibroequilibria theory with the results of direct numerical simulations in horizontally vibrated rectangular containers, finding very good agreement over a range of parameters. The calculations also reveal an important difference in the behavior between small and large fluid volumes. With dimensionless volume larger than about 0.36 , the symmetric vibroequilibria solution suffers a saddle-node instability prior to contact with the container bottom. This saddle-node bifurcation is analyzed using a simplified family of surfaces and shown to persist when gravity is included. Finally, an investigation of dynamic effects is presented, where a strong correlation is found between modulated subharmonic surface waves and the first odd sloshing mode. At large enough amplitude, this sloshing destroys the underlying vibroequilibria state and thus represents a possible instability for vibroequilibria in low viscosity fluids. Published by AIP Publishing.
\end{abstract}

\section{INTRODUCTION}

The relative ease with which a fluid moves in response to external forces is one of its defining characteristics, and the dynamics of this motion have long been the subject of both experimental and theoretical attention. An important class of problems, represented throughout nature and engineering applications, can be characterized by periodic external forcing. Such forcing can induce instability or suppress it, depending on the system and forcing parameters. It can, for example, be used to delay convection ${ }^{1}$ or the Rayleigh-Taylor instability. ${ }^{2,3}$ It can excite mean flows in the fluid bulk ${ }^{4}$ and surface waves if there is a free surface or interface to support them. ${ }^{5,6}$ These vibration-induced phenomena can affect important physical processes like mixing, or heat and mass transfer.

The generic response to vibration of a fluid mass that is not completely confined will include surface motion, but the forcing mechanism and the characteristics of the response vary with the configuration. A vertically vibrated open container with an initially flat surface presents a classic example of parametric instability. ${ }^{7}$ The trivial (flat) state loses stability at a critical forcing value to (typically) subharmonic surface waves; if the size of the container is large compared to the length scale of the instability, these waves can arrange themselves into a wide variety of patterns, depending on fluid parameters and the characteristics of the forcing function. ${ }^{8-10}$

Any orientation of the forcing other than a purely vertical one will induce immediate motion of the surface, especially near the solid walls, whose relative motion directly forces harmonic (synchronous) waves. Horizontal forcing, for example, will excite sloshing modes at low frequencies ${ }^{11-13}$ and both synchronous and subharmonic waves in wavemaker experiments at intermediate frequencies ${ }^{6,14}$ - the subharmonic waves, in fact, are due to a localized parametric forcing mechanism analogous to that of the vertical forcing case. ${ }^{15-18}$
In heterogeneous systems, such as a stably stratified layer of immiscible fluids, horizontal forcing generates a KelvinHelmholtz type instability at a critical forcing amplitude, producing a quasi-stationary undulatory surface deformation referred to as a frozen wave. ${ }^{2,19-21}$ The initial small-amplitude frozen waves can develop nonlinear profiles and more prominent oscillatory behaviour with increasing forcing, ${ }^{22}$ as well as suffering secondary instabilities like transverse amplitude modulations. ${ }^{23}$ Parametric instabilities may occur in such systems as well. ${ }^{24}$ Recent experiments in near-critical hydrogen showed that the frozen wave amplitude increases with decreasing gravity level, approaching the length scale of the container (domain) in microgravity conditions. ${ }^{25}$

Apart from the types of surface instabilities mentioned above, which exhibit a characteristic wavenumber, vibrations also induce a slow global reorientation of the fluid toward a new quasi-static equilibrium. These states, known as vibroequilibria, were observed by Faraday ${ }^{5}$ in his experiments on the flattening of fluid drops suspended beneath a vibrating plate. Like frozen waves, vibroequilibria are quasi-stationary surface configurations differing from the unforced (capillary) equilibrium and supported by the oscillatory velocity field. The spatial inhomogeneity of this velocity field, and the corresponding oscillatory pressure field, leads to deformation of the surface. In most typical situations, this deformation is negligible compared to the more familiar effects of gravity or surface tension. If the forcing is strong enough, however, vibration-induced deformation must be considered. In microgravity environments, in particular, the phenomena of vibroequilibria will be important in moderate or large systems, and may even dominate the fluid response in the absence of strong surface tension.

The theory of vibroequilibria was developed using a timeaveraged variational approach. ${ }^{26-29}$ This treatment assumes that the fluid is inviscid and the flow is irrotational so that the Lagrangian (Hamiltonian) variational method can be applied, 
and that the forcing frequency is much greater than the primary sloshing frequency. The time-averaged surface shape (i.e., the vibroequilibria) can then be found by minimizing an energy functional that includes terms accounting for gravitational energy, surface tension, and vibrational energy. ${ }^{30}$ The theory can describe compressible flows, but we concentrate here on the incompressible case, where the forcing frequency is lower than the first acoustic resonance.

Although there has been general qualitative agreement between the predictions of vibroequilibria theory and experiments, ${ }^{2,31}$ there is currently no detailed comparison, that we are aware of, between these predictions and direct numerical simulations. This paper fills this gap by comparing the predictions of the time-averaged energy functional approach with numerical simulations of fluid motion in horizontally vibrated two-dimensional rectangular containers. The vibroequilibria calculations can also be compared with those of Gavrilyuk et al. ${ }^{29}$ and Lyubimov et al. ${ }^{32}$ who noted hysteresis and coexistence between singly connected and disconnected solutions. In this paper we examine the hysteresis of vibroequilibria states in more detail, finding that, beyond a critical depth, this is related to a saddle-node instability that is present both with and without gravity.

Another potential departure from the predictions of vibroequilibria theory in real fluid systems is due to coupling with dynamic modes like surface waves and sloshing, which are especially important in low viscosity fluids. The effect of these dynamic modes on the averaged vibroequilibria state has apparently not been investigated before. We show that there can be significant interaction among these modes, depending on forcing parameters, viscosity, boundary conditions, and the initial (transient) approach to the vibroequilibria state. In particular, there is a strong association between modulated subharmonic surface waves of the type recently studied in horizontally vibrated fluid systems ${ }^{18,33-35}$ (without the vibroequilibria effect) and the first odd sloshing mode. In some cases, this odd sloshing mode grows large enough to completely disrupt the underlying vibroequilibria state.

The paper is organized as follows. In Section II we define the mathematical problem of vibroequilibria. In Section III we summarize some results on multiplicity and hysteresis in the hydrostatic (unforced) case that will be useful for interpreting subsequent vibroequilibria results. The numerical scheme used to solve the vibroequilibria problem is defined in Section IV, while results are presented in Section V for two-dimensional rectangular containers and compared with direct numerical simulations of the Navier-Stokes equations. The results reveal the instability of the vibroequilibria state for sufficiently deep containers that is analyzed in Section VI after restricting the minimization problem to specific families of surface shapes. Section VII examines the interaction of (high frequency) surface waves and sloshing modes with the average vibroequilibria state through direct numerical simulations. Conclusions are drawn in Section VIII.

\section{THE VIBRATED FLUID PROBLEM}

The problem of interest is that of an incompressible fluid, bounded by solid boundaries $W$ and a free surface $S$, and subjected to periodic acceleration $\mathbf{g}(t)$. The velocity field $\mathbf{v}$ is described by the incompressible Navier-Stokes equations,

$$
\begin{aligned}
\frac{\partial \mathbf{v}}{\partial t}+\mathbf{v} \cdot \nabla \mathbf{v} & =-\frac{1}{\rho} \nabla p+v \Delta \mathbf{v}+\mathbf{g}(t), \\
\nabla \cdot \mathbf{v} & =0,
\end{aligned}
$$

where $\rho$ is the fluid density, $v$ is the kinematic viscosity, $\nabla$ is the gradient operator, and $\Delta$ the Laplace operator. The acceleration

$$
\mathbf{g}(t)=\varepsilon \omega \cos (\omega t) \mathbf{e}_{v}-g_{0} \mathbf{e}_{z},
$$

includes vertical gravity $g_{0}$ (if present) and an applied forcing characterized by an amplitude $\varepsilon$ (with units of velocity), frequency $\omega$, and orientation along the unit vector $\mathbf{e}_{v}$.

No-slip boundary conditions apply on the solid walls while the free surface $z=f(x, y)$ satisfies the following stress balance and kinematic conditions (in a local chart),

$$
\begin{gathered}
P+\Gamma \kappa / \rho-v \hat{\mathbf{n}} \cdot\left(\nabla \mathbf{v}+\nabla \mathbf{v}^{T}\right) \cdot \hat{\mathbf{n}}=0, \\
\hat{\mathbf{n}} \times\left(\nabla \mathbf{v}+\nabla \mathbf{v}^{T}\right) \cdot \hat{\mathbf{n}}=0, \\
\frac{\partial f}{\partial t}+\bar{v} \cdot \bar{\nabla} f-v_{z}=0,
\end{gathered}
$$

where $P=p / \rho$ is the kinematic pressure [determined by Eq. (1a)], $\Gamma$ is the surface tension, $\hat{\mathbf{n}}$ is a unit vector normal to the surface, $\bar{\nabla}$ denotes the horizontal two-dimensional gradient operator, $v_{z}$ and $\bar{v}$ represent the vertical and horizontal components of velocity, and the mean curvature is given by

$$
\kappa=\frac{1}{2} \bar{\nabla} \cdot\left(\frac{\bar{\nabla} f}{\sqrt{1+|\bar{\nabla} f|^{2}}}\right) .
$$

\section{A. Variational method}

The mathematical theory of vibroequilibria ${ }^{26-29}$ makes use of the Lagrangian (Hamiltonian) variational approach to fluid mechanics.

Consider, for example, the case of an inviscid fluid with gravity $\mathbf{g}=g_{0} \mathbf{e}_{z}$. If the velocity field is potential so that $\mathbf{v}=\nabla \phi$, its compliance with the Laplace equation

$$
\Delta \phi=0,
$$

as well as the evolution of the surface,

$$
\begin{array}{r}
\frac{\partial f}{\partial t}+\bar{\nabla} \phi \cdot \bar{\nabla} f-\frac{\partial \phi}{\partial z}=0, \\
\frac{\partial \phi}{\partial t}+g_{0} f+\frac{1}{2}|\nabla \phi|^{2}-2 \kappa \frac{\Gamma}{\rho}=0,
\end{array}
$$

follows ${ }^{36}$ from eliminating first order variations in $\phi$ and $\Omega$ of the functional

$$
\begin{aligned}
\mathcal{L}= & \int_{\Omega}\left(\frac{\partial \phi}{\partial t}+g_{0} z+\frac{1}{2}|\nabla \phi|^{2}\right) d \Omega \\
& +\frac{\Gamma}{\rho} \int_{S} d S-\frac{\Gamma}{\rho} \sin \beta \int_{W} d W .
\end{aligned}
$$

The fluid domain $\Omega$ is limited by a free surface $S$ and solid walls $W$ (see Fig. 1) and the contact angle is $\pi / 2-\beta$. 


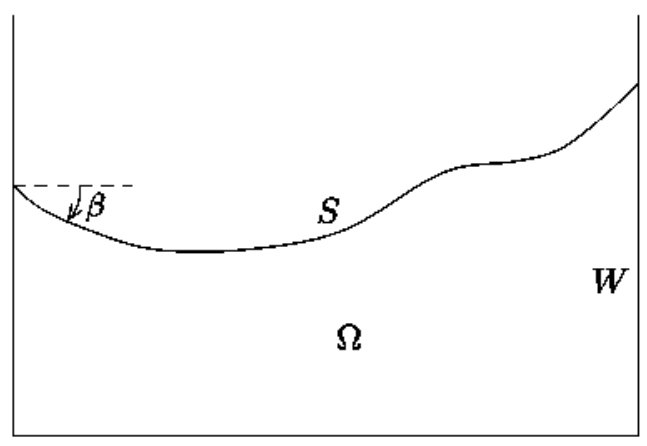

FIG. 1. Sketch of fludd domain and bounding surfaces.

\section{B. Vibroequilibria}

The theory of vibroequilibria deals with the case of high frequency periodic forcing and, in addition to assuming irrotational inviscid flow, relies on a separation of time scales between the forcing and the slower (averaged) response of the fluid. In particular, the period of the forcing should be much smaller than the dissipative time scale ${ }^{26}$ and the period of the primary sloshing modes. ${ }^{27,29}$ Incompressibility further requires that the forcing frequency is below the frequency of the first acoustic resonance.

The velocity potential is assumed, at leading order, to vary harmonically in phase with the applied high frequency forcing,

$$
\phi(\mathbf{x})=\varepsilon \bar{\phi}(\mathbf{x}) \cos (\omega t)+\cdots,
$$

where $\mathbf{x}=(x, y, z)$, while the free surface is split into an average and an oscillatory part

$$
f(x, y, t)=F(x, y)+\tilde{f}(x, y) \cos (\omega t)+\cdots .
$$

The vibroequilibria (average fluid position) characterized by $z=F(x, y)$ is dynamically stable if it is a local minimum of the averaged functional ${ }^{27,29}$

$$
\begin{aligned}
\mathcal{L}= & \int_{\langle\Omega\rangle}\left(g_{0} z+\frac{\varepsilon^{2}}{4}|\nabla \ddot{\phi}|^{2}+P_{0}\right) d \Omega \\
& +\frac{\Gamma}{\rho} \int_{\langle S\rangle} d S-\frac{\Gamma}{\rho} \sin \beta \int_{\langle W\rangle} d W,
\end{aligned}
$$

where $P_{0}$ is a Lagrange multiplier for enforcing conservation of volume. After integration by parts, Eq. (9) is equivalent to

$$
\begin{aligned}
\mathcal{L}= & \int_{\langle\Omega\rangle}\left(P_{0}+g_{0} z\right) d \Omega+\frac{\varepsilon^{2}}{4} \int_{\langle S\rangle} \tilde{\phi} \frac{\partial \tilde{\phi}}{\partial n} d S \\
& +\frac{\Gamma}{\rho} \int_{\langle S\rangle} d S-\frac{\Gamma}{\rho} \sin \beta \int_{\langle W\rangle} d W,
\end{aligned}
$$

provided $\tilde{\phi}$ satisfies the Laplace equation

$$
\Delta \tilde{\phi}=0 \quad \text { in } \quad\langle\Omega\rangle,
$$

with boundary conditions (applied in a comoving reference trame)

$$
\begin{gathered}
\frac{\partial \tilde{\phi}}{\partial n}=0 \text { on } \quad\langle W\rangle, \\
\tilde{\phi}=\mathbf{e}_{v} \cdot \mathbf{x} \text { on }\langle S\rangle .
\end{gathered}
$$

Hereafter we omit the brackets, and it should be understood that $S, W$, and $\Omega$ refer to the averaged boundaries and domain.

To obtain the vibroequilibria configuration $F(x, y)$, we must minimize the functional (10) over permissible configurations, with $\tilde{\phi}$ satistying Eqs. (11). Since $\tilde{\phi}$ on the free surface $S$ is known from Eq. (11c), it is usetul to express $q \equiv \partial \ddot{\phi} / \partial n$ on $S$ in terms of $\tilde{\phi}$ using Green's (third) identity,

$$
\begin{aligned}
\int_{S} G\left(\mathbf{x}, \mathbf{x}^{\prime}\right) q\left(\mathbf{x}^{\prime}\right) d S^{\prime}= & \int_{S} \tilde{\phi}\left(\mathbf{x}^{\prime}\right) \frac{\partial G\left(\mathbf{x}, \mathbf{x}^{\prime}\right)}{\partial n} d S^{\prime} \\
& +\int_{W} \tilde{\phi}\left(\mathbf{x}^{\prime}\right) \frac{\partial G\left(\mathbf{x}, \mathbf{x}^{\prime}\right)}{\partial n} d W^{\prime}-\frac{1}{2} \tilde{\phi}(\mathbf{x}),
\end{aligned}
$$

applied to a point $\mathbf{x}$ on the free surface $S$. The left-hand-side simplifies due to the fact that $q=0$ on the solid walls $W$. Using the fact that $\vec{\phi}=\mathbf{e}_{r^{\prime}} \cdot \mathbf{x}$ on the free surface, and taking a Green's function satisfying $\partial G\left(\mathbf{x}, \mathbf{x}^{\prime}\right) / \partial n^{\prime}=0$ on $W$ we obtain

$$
\int_{S} G\left(\mathbf{x}, \mathbf{x}^{\prime}\right) q\left(\mathbf{x}^{\prime}\right) d S^{\prime}=-\frac{1}{2} \mathbf{e}_{v} \cdot \mathbf{x}+\int_{S}\left(\mathbf{e}_{v} \cdot \mathbf{x}^{\prime}\right) \frac{\partial G\left(\mathbf{x}, \mathbf{x}^{\prime}\right)}{\partial n} d S^{\prime} .
$$

With an appropriate Green's function $G\left(\mathbf{x}, \mathbf{x}^{\prime}\right)$, this relation allows $q$ to be determined for a given surface $S$ in terms of known functions evaluated on this surface.

Assuming vertical solid walls and integrating the gravity term over $z$, we obtain

$$
\int_{\Omega} g z d \Omega=\int_{S} \frac{g}{2}\left[F(x, y)^{2}-d^{2}\right] d x d y
$$

where the bottom of the domain is located at $z=-d$ (i.e., $d$ is the depth of the fluid with a flat horizontal surface). The resulting functional involves only the boundaries of the fluid domain, $S$ and $W$, which are determined by $F(x, y)$,

$$
\begin{aligned}
\mathcal{L}= & P_{0} V+\frac{\Gamma}{\rho} \int_{S} d S+\frac{\varepsilon^{2}}{4} \int_{S}\left(\mathbf{e}_{v} \cdot \mathbf{x}\right) q(\mathbf{x}) d S \\
& -\frac{\Gamma}{\rho} \sin \beta \int_{W} d W+\frac{g_{0}}{2} \int_{S}\left[F(x, y)^{2}-d^{2}\right] d x d y,
\end{aligned}
$$

where $V$ is the fluid volume.

After nondimensionalizing the integrals in $\mathrm{Eq}$. (15) with a characteristic length $L$ (taken to be the width of the domain in what follows) and using the rescalings

$$
\mathbf{x} \rightarrow L \mathbf{x}, P_{0} \rightarrow \frac{\Gamma}{\rho L} P_{0}, \tilde{\phi} \rightarrow L \tilde{\phi}, \mathcal{L} \rightarrow \frac{\Gamma L^{2}}{\rho} \mathcal{L},
$$

we obtain

$$
\begin{aligned}
\mathcal{L}= & P_{0} \mathcal{V}+\int_{S} d S+\eta \int_{S}\left(\mathbf{e}_{v} \cdot \mathbf{x}\right) q(\mathbf{x}) d S \\
& -\sin \beta \int_{W} d W+\frac{\mathrm{B} o}{2} \int_{S}\left[F(x, y)^{2}-d^{2}\right] d x d y,
\end{aligned}
$$

where $\mathcal{V}$ is the dimensionless volume, the parameter

$$
\eta=\frac{\rho L}{4 \Gamma} \varepsilon^{2}
$$

characterizes the ratio of vibrational energy to surface energy, and the Bond number $\mathrm{B} 0=\rho g_{0} L^{2} / \Gamma$, based on the width 
$L$, characterizes the relative importance of gravitational and surface energy terms.

\section{HYDROSTATIC SOLUTIONS AND HYSTERESIS}

In the absence of gravity and forcing, the minimum energy surfaces, in two dimensions, are circular arcs consistent with the given contact angle and fluid volume. Analytical expressions for equilibrium solutions and their energies can be found. Although such solutions are well known, we present selected results here to motivate the discussion of connected and disconnected vibroequilibria solutions, which accompanies the results presented in Sections V and VI, and to emphasize the role that fluid volume plays in controlling the multiplicity of possible solutions (or lack thereof); vibrational amplitude will be seen to have a similar effect on multiplicity and hysteresis as decreasing fluid volume.

For a given domain and contact angle, there may be one or more hydrostatic solutions. For deep fluid layers in rectangular domains of length $L$ with dimensionless volume (area) $\mathcal{V}=V / L^{2}>>1$, there will be a unique, singly connected, symmetric solution $\Sigma^{c}$ that wets the bottom of the container and equal portions of the walls on either side. As volume is reduced, families of disconnected solutions arise, characterized both by the number of disconnected fluid volumes and by the relative size of each volume.

The simplest family of disconnected solutions $\Sigma_{r}^{d}$ consists of two fluid masses located at the bottom corners of the container. We can parameterize these solutions by the fraction $r$ of the total fluid volume located at the bottom left corner. Figure 2 shows several of these for $r=1$ (single fluid mass in the left corner), $r=0.75$, and $r=0.5$ (equal fluid masses) with $\beta= \pm 20^{\circ}$ and $\mathcal{V}=0.3$. The disconnected solution with equal volumes in each corner is the only one that is symmetric about the midplane $x=0$ like the singly connected solution. The case of maximal asymmetry, with a single fluid mass in one corner, is considered as the limiting case of this same family of disconnected solutions.

The energy of each solution is the sum of surface and contact energy, and these can be calculated in terms of the reduced volume $\mathcal{V}$ and the contact angle $\pi / 2-\beta$. For the symmetric connected solution the dimensionless energy, scaled by $\Gamma L$, is

$$
E^{c}=\frac{\cos \beta}{2}+\frac{\beta}{2 \sin \beta}-\sin \beta(1+2 \mathcal{V}) .
$$

This solution exists beyond a minimum volume

$$
\mathcal{V}_{\min }^{c}=\left\{\begin{array}{ll}
\frac{1}{4 \sin \beta}\left(2-\cos \beta-\frac{\beta}{\sin \beta}\right): & \beta>0 \\
\frac{1}{4 \sin \beta}\left(\cos \beta-\frac{\beta}{\sin \beta}\right) & : \quad \beta<0
\end{array} .\right.
$$

The dimensionless energy of the disconnected solutions is given by

$$
\epsilon E_{r}^{d}=2 \sqrt{\psi}(\sqrt{r}+\sqrt{1-r}) \sqrt{\mathcal{V}}
$$

where $\epsilon=\operatorname{sign}(\pi / 4-\beta)$ and

$$
\psi=\left[\frac{\pi}{4}-\beta-\sin \beta(\cos \beta-\sin \beta)\right] .
$$
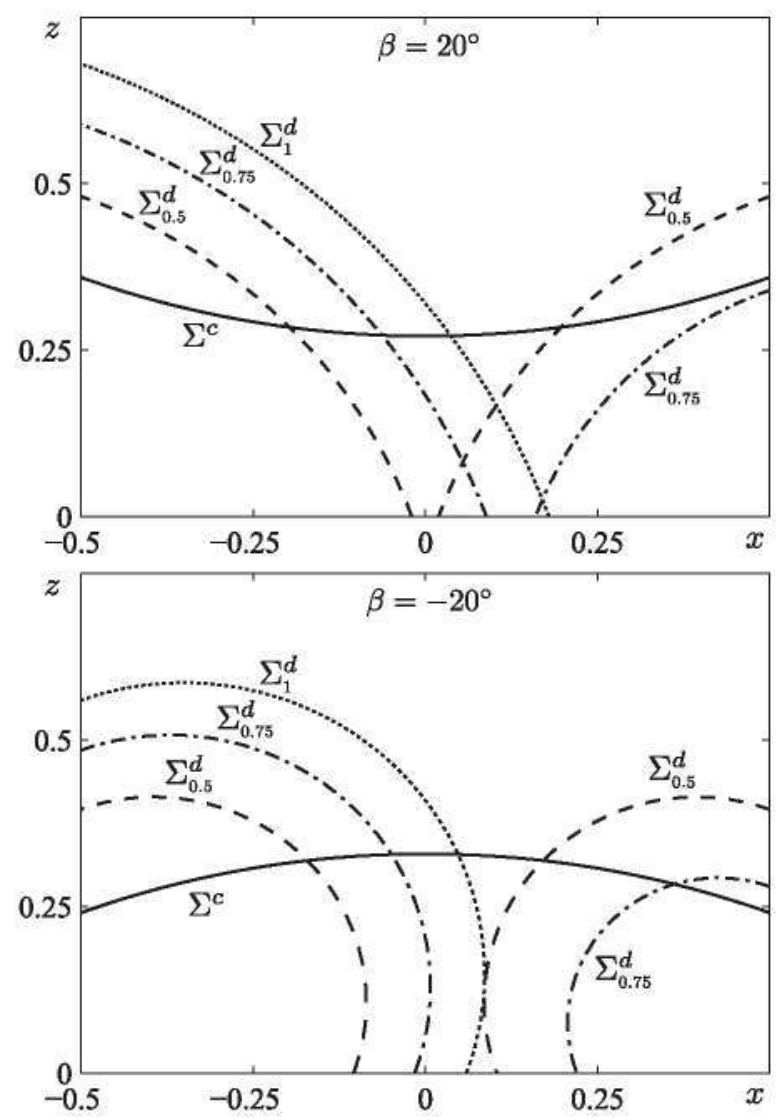

FIG. 2. Static solutions with no gravity or forcing: symmetric connected solution ( $\Sigma^{c}$ : solid curve), symmetric disconnected (two mass) solution with equal volumes $\left(\Sigma_{0.5}^{d}:\right.$ dashed curves), disconnected solution with volume ratio $0.75\left(\Sigma_{0.75}^{d}\right.$ : dotted-dashed curve), "disconnected" solution with limiting volume ratio $1\left(\Sigma_{1}^{d}\right.$ : dotted curve). Here $\beta= \pm 20^{\circ}$ (upper and lower panels, respectively) and $V / L^{2}=0.3$.

Disconnected solutions exist below the maximum volume

$$
\mathcal{V}_{\max }^{d}=\left\{\begin{array}{ll}
\frac{\psi}{(\cos \beta-\sin \beta)^{2}(\sqrt{r}+\sqrt{1-r})^{2}} & : \quad \beta>0 \\
\frac{\psi}{(1-\sin \beta)^{2}(\sqrt{r}+\sqrt{1-r})^{2}} & : \beta<0
\end{array} .\right.
$$

Figure 3 shows the energies of the connected solution $\Sigma^{c}$ and the disconnected solutions $\Sigma_{r}^{d}$, given by Eqs. (19) and (21),

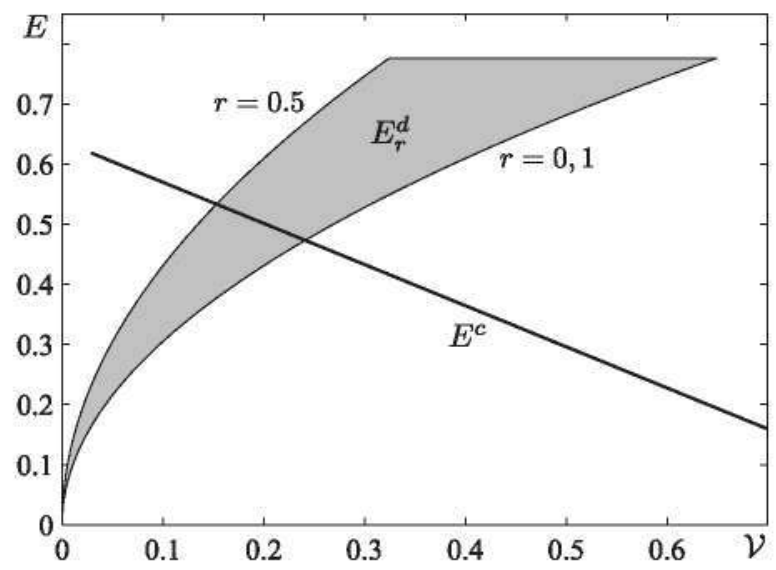

FIG. 3. Energy of static solutions with no gravity or forcing and $\beta=20^{\circ}$ : symmetric connected solution (isolated solid curve), disconnected (two mass) solutions (shaded region). 


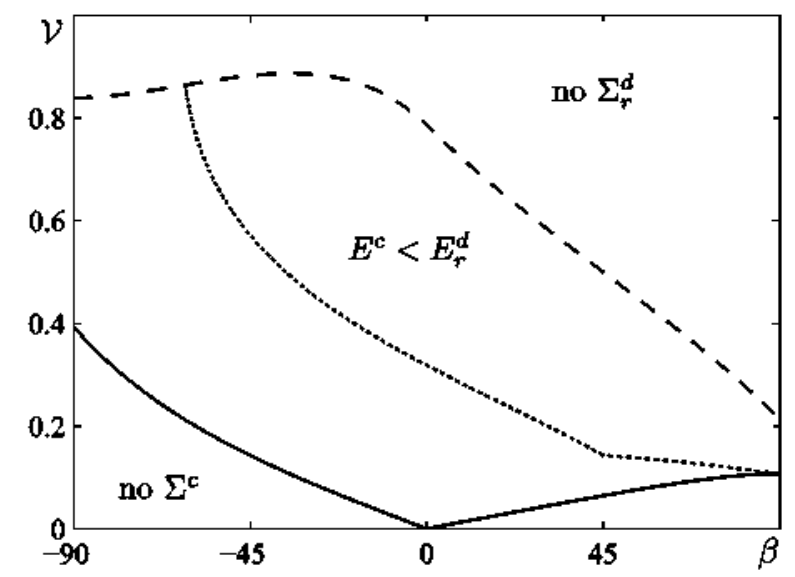

FIG. 4. Overlapping regions of existence in the $(\beta, v)$ plane with no gravity or forcing. The symmetric connected solution $\Sigma^{c}$ exists above the solid curve. Disconnected (two mass) solutions $\Sigma_{r}^{d}$ exist below the dashed curve. The dotted curve marks the point where the energy $E^{c}$ of the connected solution becomes less than that of the disconnected solutions, $E_{r}^{d}$, for all $r$.

respectively, for $\beta=20^{\circ}$. The shaded wedge-shaped region is where disconnected solutions exist. The left boundary corresponds to $r=1 / 2$ and the right boundary to $r=0,1$; the $\max$ imum energy is independent of $r$. The hysteresis illustrated here is typical of most contact angles (the region of hysteresis shrinks as $\beta \rightarrow 90^{\circ}$ ). For small volumes only the disconnected solutions exist. As $V$ increases, the singly connected solution appears, but at a higher energy than (at least some of) the disconnected solutions. In many cases, like $\beta=20^{\circ}$, this connected solution becomes the global energy minimum at a value of $\mathcal{V}$ where many disconnected solutions still exist. In other cases $\left(\beta<-61.6^{\circ}\right)$ this connected solution only becomes the global minimum as the last disconnected solution with $r=0,1$ ceases to exist at $\mathcal{V}_{\max }^{\prime}$ given by Eq. (23).

The regions where the connected and disconnected solutions exist in the $(\beta, \mathcal{V})$ plane are shown in Fig. 4, which confirms that the scenario described for $\beta=20^{\circ}$ is typical for $-61.6^{\circ}<\beta$. For all contact angles, however, there is a region (between the solid and dashed curves) where multiple solutions coexist. Although solutions will be modified when forcing (or gravity) is included, a region of multiplicity will generally remain, and transitions among these solutions can be expected to play an important role in the dynamics of the forced problem.

\section{VIBROEQUILIBRIA PROBLEM: NUMERICAL SOLUTION}

Vibroequilibria in the forced problem are found by minimizing the functional (17) over permitted surface configurations described by $F(x, y)$. Generally, one must discretize $F(x, y)$ or approximate it with a truncated expansion using suitable basis functions. Here we explore the dependence of vibroequilibria on important parameters by performing a large number of computations and, for the sake of feasibility, only two-dimensional domains are considered. The free surface $S$ is thus described by a curve $z=F(x)$ (possibly composed of disconnected segments) that can be discretized with $N$ elements of length $l_{i}=\sqrt{\left(x_{i}-x_{i-1}\right)^{2}+\left(z_{i}-z_{i-1}\right)^{2}}$.
The discretized functional (17) becomes

$$
\begin{aligned}
\mathcal{L} \simeq & P_{0} \sum_{i}^{N} l_{i} z_{i}^{m}+\sum_{i}^{N} l_{i}-\sin \beta\left(z_{N}+z_{1}+1\right) \\
& +\eta \sum_{i}^{N} \mathbf{e}_{i^{r}} \cdot\left(x_{i}^{m}, z_{i}^{m}\right) l_{i} q_{i} \\
& +\frac{\mathrm{B} 0}{2}\left[\sum_{i}^{N}\left(x_{i}-x_{i-1}\right)\left(\frac{z_{i}+z_{i-1}}{2}\right)^{2}-d^{2}\right] .
\end{aligned}
$$

A superscript $m$ means that the quantity is evaluated at the midpoint of the element, and we approximate $V_{i} \simeq l_{i} z_{i}^{m}$. With the rescaling (16), the length of the fixed bottom of the container is unity.

For the boundary element method we assume, for simplicity, that $q_{i}$ is constant over each element. Green's identity is evaluated at the center point of each surface element, as is the potential (a first order Gauss quadrature). The problem may be written as a linear system

$$
\sum_{j} K_{i j} q_{j}=\sum_{j} H_{i j}\left(\mathbf{e}_{*} \cdot \mathbf{x}_{j}^{m}\right)
$$

where the lowest order quadrature is taken for all but the diagonal elements

$$
\begin{gathered}
K_{i j} \simeq l_{j} G\left(\mathbf{x}_{i}^{m}, \mathbf{x}_{j}^{m}\right), \\
H_{i j} \simeq l_{j} \frac{\partial G\left(\mathbf{x}_{i}^{n}, \mathbf{x}_{j}^{m}\right)}{\partial n_{j}},
\end{gathered}
$$

for $i \neq j$. The diagonal terms $H_{i j}$ can be obtained from the condition $\sum_{j} H_{i j}=0$ (constant potential implies zero normal flow), while the diagonal terms $K_{i j}$ contain singularities that must be removed for each particular Green's function.

For connected solutions we use the Green `s function

$$
\begin{aligned}
& G\left(w ; w^{\prime}\right)=\frac{1}{4 \pi} \ln \left|w-w^{\prime}\right|^{2}+\frac{1}{4 \pi} \ln \left|w-\bar{w}^{\prime}\right|^{2}, \\
& \text { with } \quad w=\cos (\pi[x-1 / 2+i(y+d)]),
\end{aligned}
$$

which fulfills $\partial G / \partial n^{\prime}=0$ on all of the fixed walls. An overbar here denotes the complex conjugate. After expanding about the singularity,

$$
K_{i i} \simeq \frac{l_{i}}{2 \pi}\left[\ln \left(\frac{l_{i} \pi}{2}\left|\sqrt{1-\left(w_{i}^{m}\right)^{2}}\right|\left|w_{i}^{m}-\bar{w}_{i}^{m}\right|\right)-1\right] .
$$

Choosing equally spaced, fixed ordinates $x_{i}$ yields a functional $\mathcal{L}\left\{z_{i}\right\}$ depending only on the vertical displacements, a suitable target for minimization.

For the disconnected solutions, describing separate fluid masses attached to the corners, the following Green's function is used:

$$
\begin{aligned}
G\left(w ; w^{\prime}\right)= & \frac{1}{4 \pi}\left(\ln \left|w-w^{\prime}\right|^{2}+\ln \left|w-\bar{w}^{\prime}\right|^{2}\right. \\
& \left.+\ln \left|w+w^{\prime}\right|^{2}+\ln \left|w+\bar{w}^{\prime}\right|^{2}\right),
\end{aligned}
$$

with $\quad w=x+i y$.

As an alternative to the discretization described above, the surface may be expanded in terms of basis functions $P_{n}(x)$,

$$
F(x)=\sum_{i} a_{n} P_{n}(x)
$$


which yields a functional $\mathcal{L}\left\{a_{n}\right\}$ depending on a finite set of coefficients $a_{n}$. This approach, with Legendre polynomials for $P_{n}(x)$, was found to be both faster and more robust numerically and, thus, is the method used to obtain most of the results reported below.

\section{VIBROEQUILIBRIA RESULTS AND COMPARISON WITH NAVIER-STOKES SIMULATIONS}

Here we present the results obtained using the vibroequilibria potential theory outlined above and compare some of these with the results of direct numerical simulations of the Navier-Stokes equations. This is an important test because viscosity and damping, neglected in the vibroequilibria theory, are known to be important in determining streaming flow, ${ }^{37}$ and they should therefore affect the (quasi-stationary) equilibrium reached by the fluid.

The vibroequilibria are calculated, using either the discretized surface points $\mathbf{x}_{i}$ or the Legendre polynomial expansion of Eq. (30), in Matlab ${ }^{38}$ with the subroutine fmincon. The $P_{0} V$ term in Eq. (15) is implemented as a constraint to maintain constant volume and is not directly included in the minimization process.

To initialize the vibroequilibria calculations we use the following procedure. Symmetric connected solutions are located by allowing an initially flat (horizontal) surface to relax to the hydrostatic solution of Section III, then turning on the vibrational forcing and minimizing the functional (17). Disconnected solutions are found in analogous fashion, first allowing (right isosceles) triangular domains in each corner to relax to the hydrostatic solutions, then minimizing the functional (17) with forcing included. The initial minimization process takes only a few seconds on a typical $\mathrm{PC}$, depending on the resolution.

COMSOL Multiphysics ${ }^{39}$ is used for the direct numerical simulations with a weak, Arbitrary Lagrangian Eulerian (ALE) formulation of the Navier-Stokes equations. These simulations begin from a flat (horizontal) surface.

Figure 5 shows that the surface shape calculated using vibroequilibria theory and that found in the numerical simulation agree very well for moderate forcing amplitude. Here the Navier-Stokes simulations are performed with a fluid volume (area) $V=0.8 \mathrm{~cm}^{2}$ in a domain of width $L=2 \mathrm{~cm}$ vibrated at $50 \mathrm{~Hz}$ with amplitude $\varepsilon=3.97 \mathrm{~cm} / \mathrm{s}$. The fluid parameters used were density $\rho=1 \mathrm{~g} / \mathrm{cm}^{2}$, (one-dimensional) surface tension $\Gamma=20$ dyn (characteristic of silicone oils), and viscosity $v=200 \mathrm{cSt}$. The dimensionless volume and forcing were $V=0.2$ and $\eta=0.394$; gravity was not considered $(\mathrm{B} 0=0)$. The use of the relatively high kinematic viscosity of $200 \mathrm{cSt}$ in the simulation of Fig. 5 means that surface wave phenomena, which will be examined later, are minimal here, although small harmonic surface waves are indeed present near the boundaries.

When the forcing amplitude is varied, with the remaining parameters as in Fig. 5, the excellent agreement between vibroequilibria theory and numerical simulations holds over the entire range of forcing values considered, as seen in Fig. 6 . At a forcing of $\eta \simeq 2.75$, when the dimensionless height of the midpoint is about 0.02 , the numerical simulation fails as the

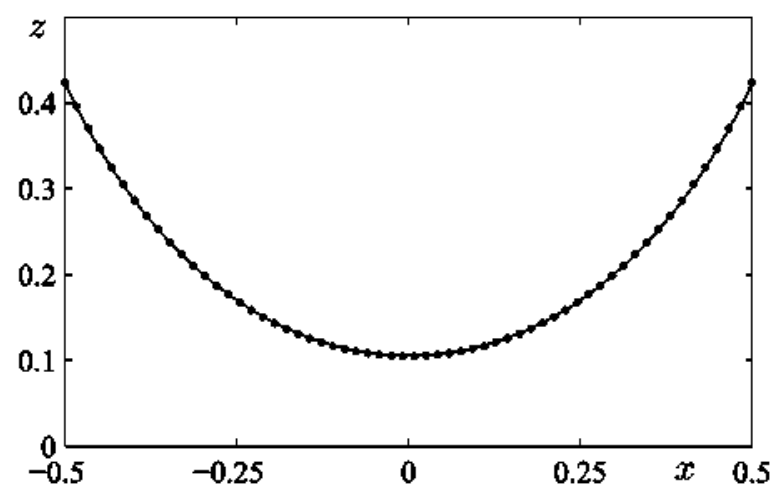

FIG. 5. Compatison of fluid surface obtained from vibroeguilibria formulation (solid dots) and Navier-Stokes simulations (continuous curve) without gravity. Paraneters are $V=0.8 \mathrm{~cm}^{2}, \beta=60^{\circ} . L=2 \mathrm{~cm} . \rho=1 \mathrm{~g}^{\prime} \mathrm{cm}^{2}$, $\Gamma=20 \mathrm{dyn}, v=200 \mathrm{cSt}, \varepsilon=3.97 \mathrm{~cm} / \mathrm{s}$, and $\omega / 2 \pi=50 \mathrm{~Hz}$, with the corresponding dimensionless parameters $\dot{v}=0.2, \eta=0.394$, and $\mathrm{Bo}=0$.

fluid nears the lower boundary. Vibroequilibria theory, however, indicates that the fluid smoothly reaches the bottom of the container at $\eta=3,30$. The results of the numerical simulations were obtained by linearly increasing the forcing velocity $\varepsilon$ at a rate of $0.031 \mathrm{~cm} / \mathrm{s}^{2}$.

Figure 7 shows how horizontal vibrations can cause the surface to deform smoothly until reaching the bottom of the container. This is not always the case. If the container is deep enough, there is a critical vibrational amplitude where the surface suffers an instability before reaching the bottom. At this point the (central part of the) surface plunges toward the bottom of the container and the fluid forcefully splits into two (or more) disconnected volumes. This instability is reflected in the eigenvalues of the Hessian of the functional (17), the smallest of which tends to zero as this point is approached. Figure 8 shows the behavior of this eigenvalue (calculated numerically) and the critical surface at the point of instability.

Figure 9 shows the dependence of this instability on $v$ for several different contact angles by recording the fluid depth (height above the bottom of the container) at the midpoint of the symmetric connected solution at the highest forcing value

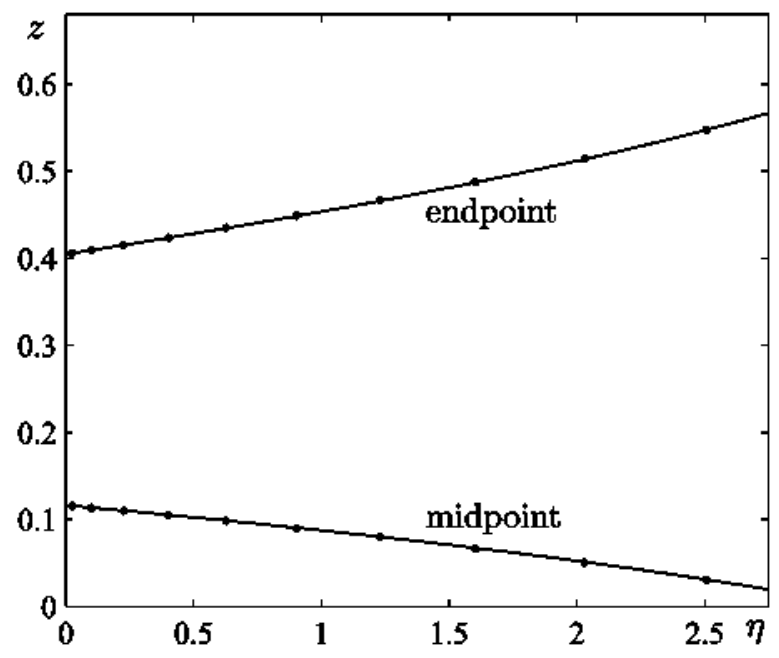

FIG. 6. Compatison of the (averaged) contact point and midpoint of the sutface obtained from vibtoecuilibtia formulation (solid dots) and Naviet-Stokes simulations (continuous curve) without gravity as a function of the vibrational forcing amplitude $\eta$. Remaining parameters are as in Fig. 5. 


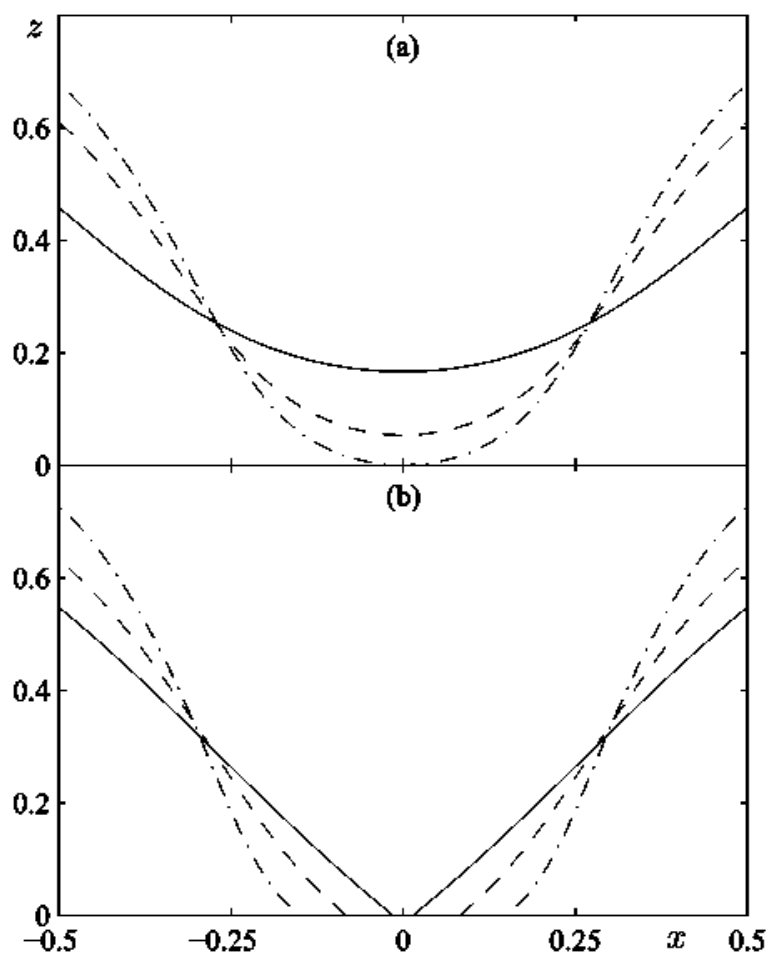

FIG. 7. Surfaces calculated using vibroequilibtia theory. At all three forcing values both (a) connected and (b) disconnected solutions exist simultaneously. Parametets are: $\nu=0.265, \beta=45^{\circ}$, and $\mathrm{Bo}=0$, with forcing values of $\eta=2.19$ (solid curves) $\eta=8.76$ (dashed curves) and $\eta=19.71$ (dasheddotted curves).

for which a corresponding minimum of $\mathrm{Eq}$. (17) can be found, a quantity denoted by $\delta_{\min }$. At this point, either the symmetric connected solution touches the bottom or the calculation
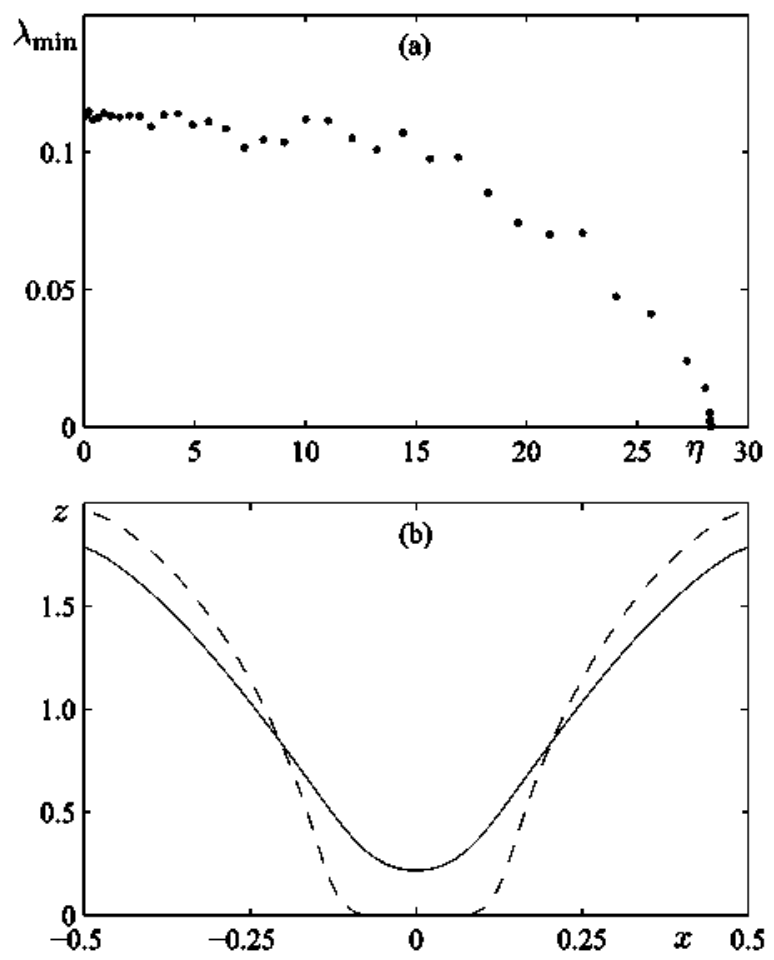

FIG. 8. With $\eta=1$ there is an instability at $\eta=28.3$. (a) Behavior of the most dangerous eigenvalue of the Hessian. (b) Critical surface just prior to instability (solid curve) and the disconnected solution (dashed curve) that also exists at this forcing value. Remaining parameters are: $\beta=45^{\circ}$ and $\mathrm{Bo}=0$.

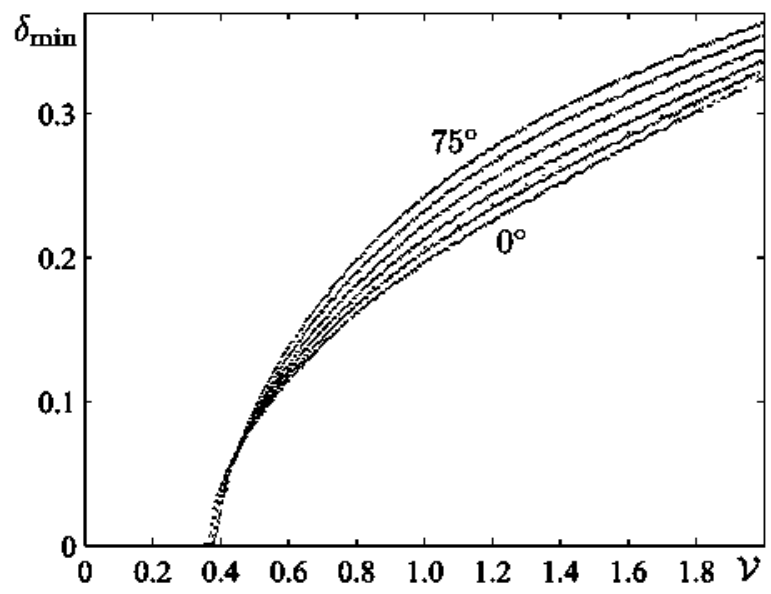

FIG. 9. Minimum depth $\delta_{\min }$ of the midpoint of the connected solution versus $\nu$ for $\mathrm{Bo}=0$ and distinct values of $\beta$ (noving upward through the curves for $v \geq 0.45): 0^{\prime}, 15^{\circ}, 30^{\circ}, 45^{\circ}, 60^{\circ}, 75^{\prime \prime}$.

fails due to the instability shown in Fig. 8. The dependence of $\delta_{\text {min }}$ on $\mathcal{V}$ reveals two regimes: for $\mathcal{V} \lesssim 0.36$, the surface of the connected vibroequilibria solution smoothly dips toward the

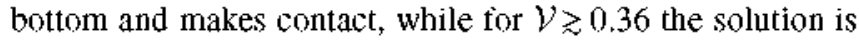
disrupted prior to this by an instability.

Note that this instability depends only weakly on contact angle. Varying $\beta$ between $0^{\circ}$ and $75^{\circ}$ only alters the boundary of the instability regime by about $4 \%$; the critical value of $\mathcal{V}$ above which the instability occurs is about 0.36 for $\beta=0^{\circ}$ and 0.375 for $\beta=75^{\circ}$.

\section{A. Multiplicity of solutions}

When the symmetric connected solution either loses stability (in sufficiently deep containers) or is destroyed by contact with the container bottom (in shallow containers) the fluid selects a distinct configuration that is, in general, disconnected. As in the hydrostatic case, there are an infinite number of disconnected solutions available, characterized by the number and volume of the separate fluid masses. Each of these disconnected solutions will exist for forcing values beyond a certain lower bound (possibly zero) where two (or more) fluid masses make contact with each other.

Figure 10 shows that, at the point of instability or contact with the container bottom, there are typically an infinite number of asymmetric disconnected solutions available at lower energy (cf. Fig. 3). Just as in the hydrostatic case with decreasing volume, the fluid, which is gradually separated and "pushed" toward the lateral walls by increasing horizontal vibration, must select one of these disconnected solutions. The variational approach does not provide selection criteria for this process, which is generally dynamic.

If the container is not too deep, as in Fig. 10(a), then the surface of the symmetric connected solution smoothly approaches the container bottom (at the midpoint) with increasing forcing. After contact is made (tangentially), there must be an adjustment of the fluid surfaces to conform with the given contact angle, and this can be associated with a sudden separation of the fluid (rapid motion of the contact points along the container bottom). Splashing may occur but, if not, it can be expected that the fluid will separate into two volumes of equal size and shape. These fluid volumes press 

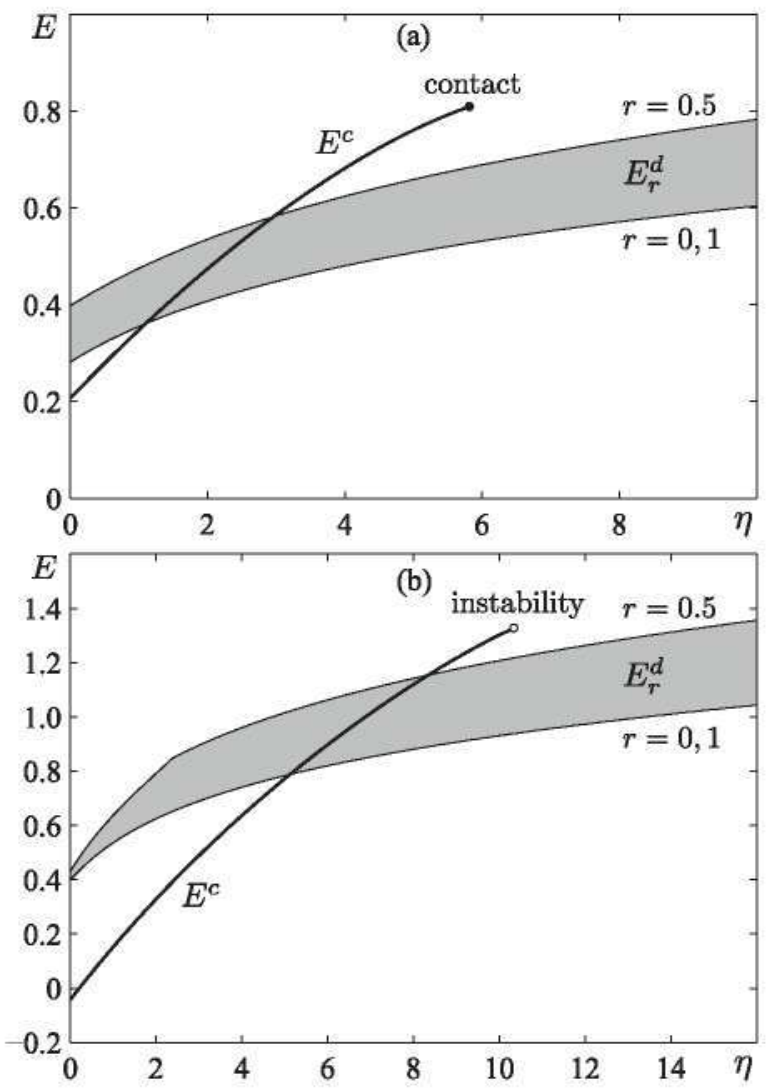

FIG. 10. Dimensionless energies of the symmetric connected solution $\left(E^{c}\right.$, solid curve) and of two disconnected fluid masses ( $E_{r}^{d}$, shaded region) parameterized by the ratio $r$ of their respective fluid volumes. The vibroequilibria calculations are done with $\beta=30^{\circ}$ and $\mathrm{Bo}=0$. For (a) $\nu=0.25$ the fluid smoothly touches the bottom at $\eta=5.79$ while for (b) $\nu=0.5$ the symmetric connected solution first suffers an instability at $\eta=10.24$.

more closely against the horizontal walls as vibration amplitude is increased. Despite its likely selection in the case of an orderly separation process, this symmetric disconnected solution is of higher energy than the asymmetric solutions that exist simultaneously.

If the container is deep enough, as in Fig. 10(b), then the symmetric connected solution loses stability prior to contact with the container bottom. Again, there will be a rapid (nonlinear) selection process as the fluid moves toward a disconnected solution, and this can be expected to be more violent and disordered than in the case of gradual contact. Splashing and sloshing are more likely to occur due to the velocity acquired by the fluid as the surface plunges toward the container bottom and crashes against it. The disordered selection process that follows is likely to amplify existing asymmetries and to increase the likelihood of asymmetric disconnected solutions being found as the final configuration. At least in the case of two disconnected fluid volumes, the asymmetric solutions are of lower energy than the symmetric one.

\section{INSTABILITY OF CONNECTED SOLUTION}

The results in Sec. V show that increasing horizontal vibration, which acts to push fluid toward the vertical walls and out of the middle of the container, is analogous to decreasing volume in the static case. Eventually the continuous symmetric solution is destroyed and the fluid must separate; the simplest separation creates two (symmetric) fluid masses located at opposite corners of the recipient.

In the hydrostatic case of Section III, the destruction of the symmetric connected solution as volume is decreased is always via contact with the bottom of the container. Until this breaking point is reached, the minimum height of the fluid, measured at the midpoint of the container for $\beta>0$, decreases smoothly (linearly, in fact) with decreasing volume.

In the case of a vibrated fluid, the destruction of the symmetric connected solution as forcing amplitude increases may be either smooth or sudden, depending on fluid volume. For $\mathcal{V} \lesssim 0.36$ the fluid surface smoothly approaches the bottom of the container but for larger $\mathcal{V}$ the symmetric connected solution suffers an instability prior to contact. As discussed above, at this point the vibroequilibria surface plunges toward the bottom, which suggests a more violent and unpredictable separation process than in the hydrostatic case.

We analyze the nature of this instability in this section by first simplifying the variational problem. Rather than minimizing the functional $\mathcal{L}$ of Eq. (17) over all possible surface configurations, we select two families of surfaces $z=h(x)$ that are qualitatively similar to the calculated surfaces [see Figs. 7(a) and 8(b)]. We take, for simplicity, the case of $\beta=0^{\circ}$ (contact angle $90^{\circ}$ ). Each of the surfaces, shown in Fig. 11, has zero mean (to preserve volume), and is parameterized by a single amplitude $\alpha$.

\section{A. Without gravity}

With a one-parameter family of surfaces like those shown in Fig. 11, the variational problem of Eq. (17) with $\mathrm{Bo}=0$ simplifies to that of finding critical points of the function

$$
\mathcal{L}(\alpha ; \eta)=P_{0} \mathcal{V}+E_{\text {sur }}(\alpha)+\eta E_{\text {vib }}(\alpha),
$$

where $E_{\text {sur }}$ denotes the surface energy (including contact energy) and $E_{\text {vib }}$ is the (normalized) vibrational energy,

$$
\begin{aligned}
& E_{\text {sur }}=\int_{S} d S-\sin \beta \int_{W} d W, \\
& E_{\text {vib }}=\int_{S}\left(\mathbf{e}_{v} \cdot \mathbf{x}\right) q(\mathbf{x}) d S .
\end{aligned}
$$

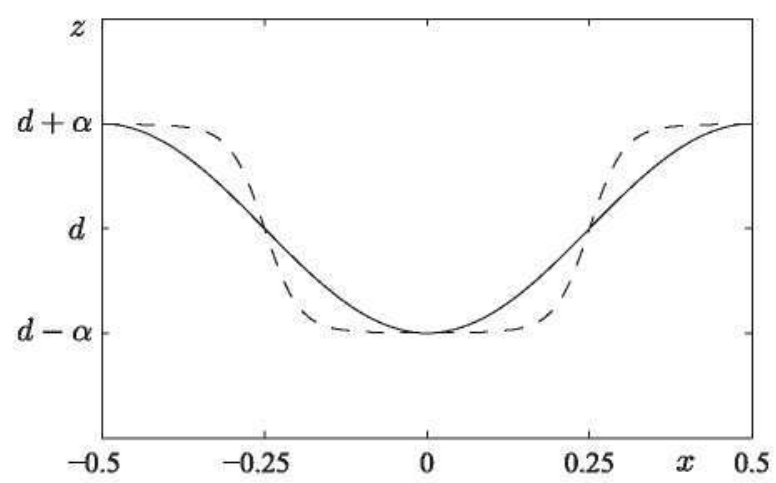

FIG. 11. Solution families used to approximate the vibroequilibria: a simple cosine function $z=d+\alpha \cos (2 \pi x+\pi)$ (solid curve) and a hyperbolic tangent profile $z=d+\alpha \tanh [3 \cos (2 \pi x+\pi)]$ (dashed curve) describing a steeper dip in the middle, as observed with higher forcing values. Here $d$ is the dimensionless depth of the undisturbed surface (equal to $v$ for rectangular containers). 
The selected surface is an extremum of $\mathcal{L}$ with respect to $\alpha$ so

$$
E_{\text {sur }}^{\prime}(\alpha)=-\eta E_{\mathrm{vib}}^{\prime}(\alpha)
$$

where the prime denotes a derivative in $\alpha$. In other words, the variation with $\alpha$ of the surface energy and vibrational energy must cancel for the total energy $E=E_{\text {sur }}+\eta E_{\mathrm{vib}}$ to have a critical point. It is easy to visualize the critical surface amplitude by plotting these difterent energies.

For deep containers, both families of surfaces (see Fig. 11) exhibit two critical points up to a certain forcing value. The critical point at smaller $\alpha$ is an energy minimum (stable), while the critical point at larger $\alpha$ is an energy maximum (unstable). As forcing $\eta$ is increased, these two critical points approach each other and merge in a saddle-node bifurcation that eliminates the symmetric connected solution. Figure 12 illustrates this process for the cosine surface profile. Results for the hyperbolic tangent surface profile are similar.

The symmetric connected solution reaches its minimum depth $\delta$ at the midpoint of the surface, and we use this quantity to characterize the critical points in Fig. 13. For both the cosine and hyperbolic tangent surface families, the qualitative picture is the same. For shallow containers (like $\mathcal{V}=0.1,0.25$ ) the minimum depth $\delta$ decreases smoothly to zero as forcing $\eta$ is increased (indicating contact with the container bottom). For deeper containers there is a turning point where the (local) minimum energy solution at smaller $\alpha$ joins with a (local) maximum energy solution at larger $\alpha$ in a saddle-node bifurcation.

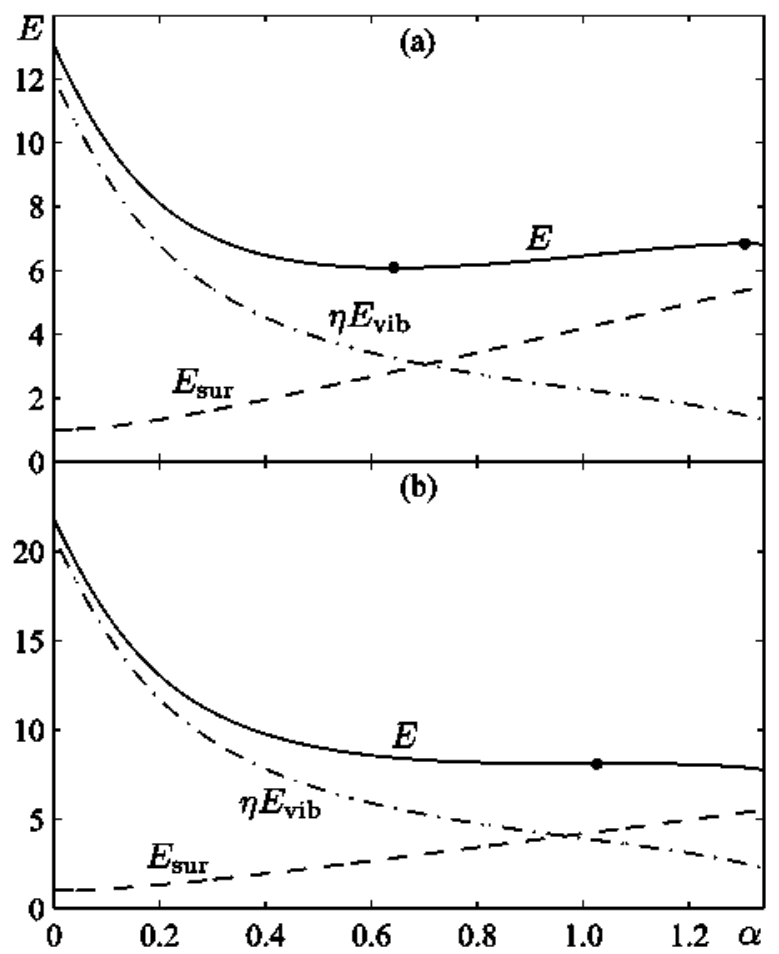

FIG. 12. Surface ( $E_{\text {sur. }}$ dashed curve), vibrational ( $\eta E_{\text {qib. }}$, dashed-dotted curve), and total energies ( $E$, solid curve) for the cosine family of surfaces with $v=1.35$ (deep container) as a function of $\alpha$. (a) Two critical points (shown with solid dots) with $\eta=44.3$, one stable at $\alpha=0.642$ and one unstable at $\alpha=1.305$. (b) Saddle-node bifurcation at $\eta=76.7$ where the two critical points merge at $\alpha=1.025$.

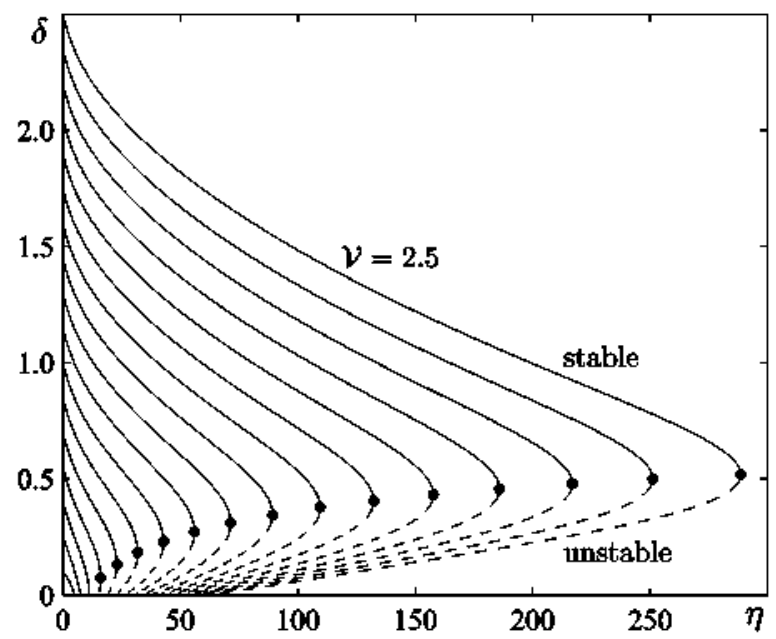

FIG. 13. Minimum depth $\delta$ of the critical surfaces as a function of $\eta$ for the cosine family of surfaces. The curves shown are for volumes $v=0.1$, $0.25, \ldots, 2.35,2.5$, increasing ontward in steps of 0.15 . For $v$ values with a satdle-node bifurcation (marked with solid dots), the upper solutions (solid curves) are stable while the lower solutions (dashed curves) are unstable.

Beyond this forcing value, which increases with $\mathcal{V}$, the symmetric connected solution vanishes and the fluid must find a disconnected vibroequilibria solution.

We confirm that this scenario of a stable symmetric connected solution colliding with an unstable (larger amplitude) solution and disappearing in a saddle-node bifurcation is correct for sufficiently large $\mathcal{V}$ by comparing the calculations based on the simple one-parameter family of surfaces with the vibroequilibria calculations that permit any (smooth) surface. Figure 14 shows that the approximation based on the cosine family of surfaces is surprisingly good at capturing the dependence of $\delta$ on $\eta$ and $\nu$. As one can see in Fig. 15, the cosine family of surfaces overestimates the lower bound of the saddlenode bifurcation somewhat, placing it at $\nu=0.455$, while the hyperbolic tangent family underestimates it, at $\mathcal{V}=0.305$. Despite such differences, both one-parameter families clearly predict the same qualitative dependence of $\delta_{\min }$ on $\mathcal{V}$ seen in

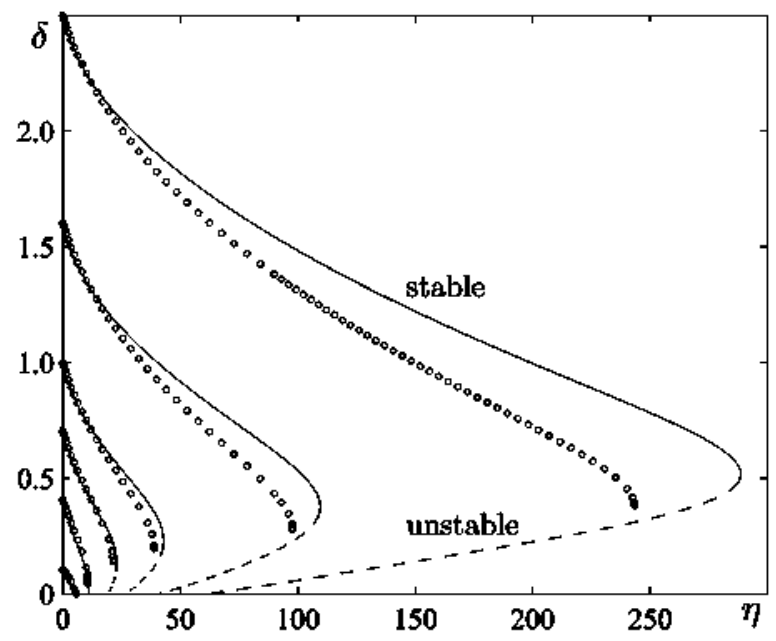

FIG. 14. Comparison of $\delta$ for the critical surfaces of the cosine solution family (solid curves stable, dashed curves unstable) with that obtained from the full vibroequilibtia calculation (open circles) on the stable branch. The volumes (equivalently, depths) used are $v=0.1,0.4,0.7,1,1.6,2.5$ (increasing outward from the origin). 


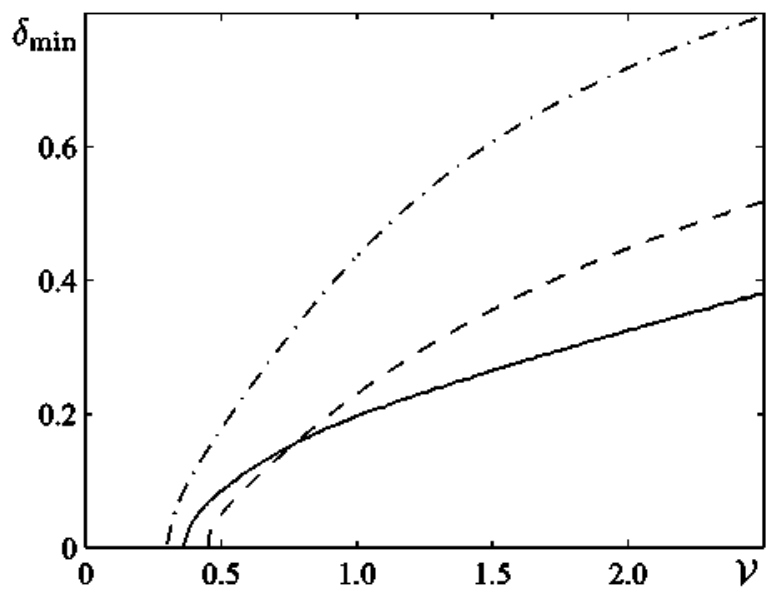

FIG. 15. Comparison of $\delta_{\min }$ (value of $\delta$ at the saddle-node bifurcation) obtained with the full vibroequilibria calculation (solid curve), the cosine family (dashed curve), and the hyperbolic tangent family (dashed-dotted curve). The lower bound of the saddle-node bifurcation is $v=0.36,0.455 .0 .305$ in these three cases. respectively.

Section V. This strongly suggests that such behavior is generic and not dependent on the details of the approximation used here to describe the vibroequilibria surface.

\section{B. With gravity}

With gravity present, the variational problem of $\mathrm{Eq} .(17)$, restricted to a one-parameter family of surfaces as in Fig. 11, simplifies to that of locating extrema of the function

$$
\mathcal{L}(\alpha ; \eta)=P_{0} \mathcal{V}+E_{\text {sur }}(\alpha)+\eta E_{\mathrm{vib}}(\alpha)+E_{g}(\alpha),
$$

where $E_{\text {sur }}$ and $E_{\text {vib }}$ are again given by Eqs. (32) and (33), respectively, and the gravitational energy is

$$
E_{\mathrm{g}}=\frac{\mathrm{B} 0}{2} \int_{S}\left[F(x, \alpha)^{2}-d^{2}\right] d x .
$$

The derivative of $\mathcal{L}$ with respect to $\alpha$ must vanish for a critical surface,

$$
0=E_{\text {sur }}^{\prime}(\alpha)+\eta E_{\text {vib }}^{\prime}(\alpha)+E_{g}^{\prime}(\alpha)
$$

meaning that the variations of surface, vibrational, and gravitational energy cancel out.

Figure 16 illustrates how these three types of energy vary with the "vibroequilibria amplitude" $\alpha$. For deep enough containers the same scenario occurs as in Section VI A. Initially, with very small forcing $\eta$, there is a unique stable connected solution that deviates very little from the hydrostatic surface. As forcing is increased, this solution grows in amplitude, dipping further in the center of the container and moving upward along the vertical walls. At a certain value of $\eta$, a second unstable solution arises; its surface just reaches the bottom of the container, and it is characterized by a larger amplitude $\alpha$. With increasing forcing, this second unstable solution diminishes in size, moving upward in the center and downward along the vertical walls. The two solutions, the first a local energy minimum and the second a local energy maximum, move towards each other as $\eta$ is further increased and finally merge in a saddle-node bifurcation. Beyond this saddle-node point, there are no symmetric connected solutions.

Note that, although gravitational energy typically dominates over surface energy for moderately sized containers

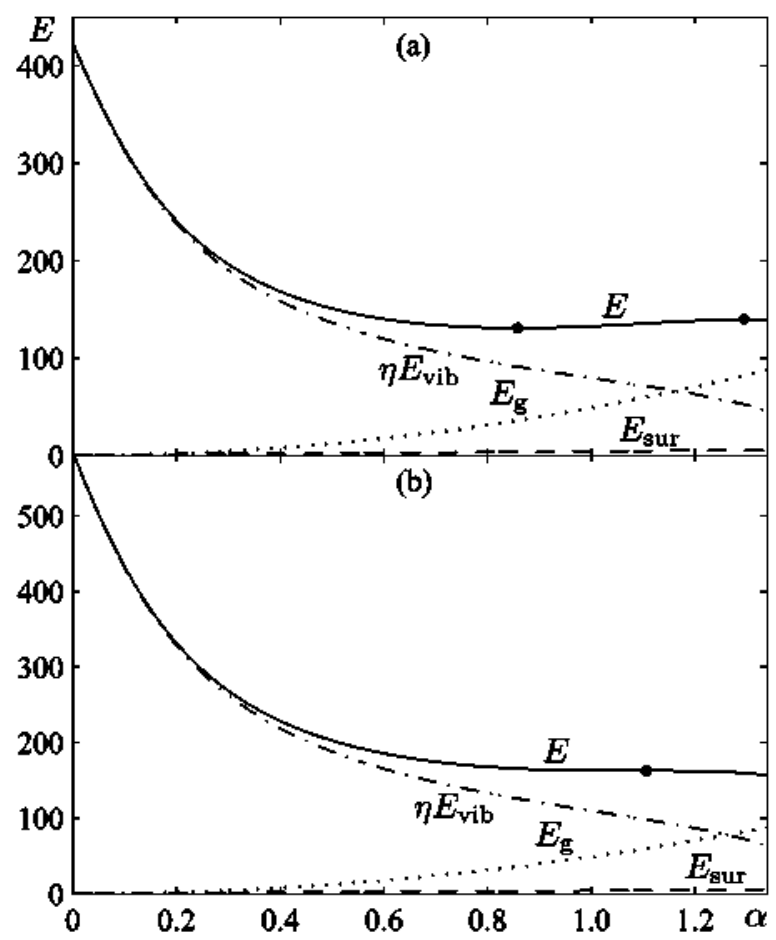

FIG. 16. Surface ( $E_{\text {sur }}$, dashed curve), gravitational ( $E_{\mathrm{g}}$, dotted curve), vibrational ( $\eta E_{\text {vib }}$, dashed-dotted curve), and total energies ( $E$, solid curve) for the cosine family of surfaces with $v=1.35$ (deep container) as a function of $\alpha$. (a) Two critical points (shown with solid dots) with $\eta=1557$, one stable at $\alpha=0.858$ and one unstable at $\alpha=1.295$. (b) Saddle-node bifurcation at $\eta=2150$ where the two critical points merge at $\alpha=1.106$. The Bond number Bo $=196$.

(Bo $=196$ in the calculations of Fig. 16), it also increases with amplitude $\alpha$ and so plays a similar role. The main effect of adding gravity, compared to the case of $\mathrm{Bo}=0$ in Fig. 12, is that much larger forcing values are required to see the equivalent vibroequilibria phenomena when Bo is large. As in the case of $\mathrm{Bo}=0$, there is a minimum volume $(\mathcal{V} \sim 0.6)$ beyond which the saddle-node point exists.

With gravity, the simple cosine and hyperbolic tangent families of surfaces provide a less accurate quantitative

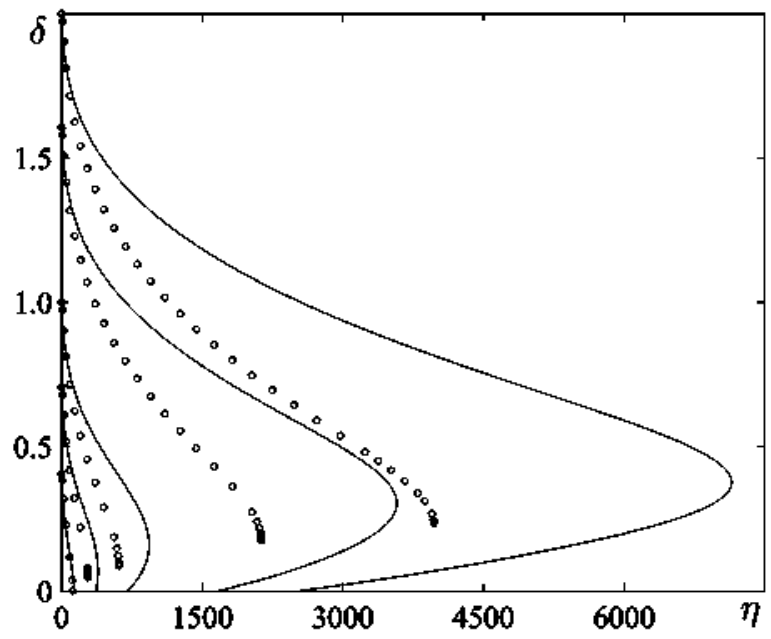

FIG. 17. Conparison of $\delta$ for the critical surfaces of the cosine solution family (solid curves) with that obtained from the full vibroequilibria calculation (open circles) on the stable branch. The volumes used are $v=0.4,0.7,1,1.6,2$ (increasing outward from the origin) and $\mathrm{Bo}=196$. 
comparison with the full vibroequilibria calculations. In particular, they tend to overestimate the forcing value of the saddle-node bifurcation, as seen in Fig. 17 for the cosine surface family. Despite this, it is clear that the basic qualitative scenario is still captured and, thus, that a saddle-node bifurcation is implicated both with and without gravity.

\section{SURFACE WAVES AND DYNAMICS}

The numerical simulations of the Navier-Stokes equations used in Figs. 5 and 6 of Section V were performed with high viscosity fluid $(200 \mathrm{cSt})$ in order to suppress surface waves and their effects. In this section we look at the case where larger amplitude surface waves are present and investigate the interaction between them and the vibroequilibria (average) surface.

With low-viscosity fluids, surface waves are supported with less forcing amplitude and penetrate further into the interior of the container. Figure 18 shows a comparison of the surface predicted by vibroequilibria theory with that obtained by direct numerical simulation using the same forcing amplitude and viscosities of $5 \mathrm{cSt}$ and $10 \mathrm{cSt}$. The simulations are done in a container of length $9 \mathrm{~cm}$ with a fluid volume of $45 \mathrm{~cm}^{2}$ and surface tension appropriate for silicone oil, and parameters that correspond to recent surface wave experiments. ${ }^{18,40}$ Although the (vibroequilibria) surface here is not flat, similarities to the surface wave dynamics of the flat case may be anticipated, including temporal modulation of the subharmonic waves. ${ }^{18,33,35}$

With viscosity $v=10 \mathrm{cSt}$ the surface waves seen in the simulations are of modest amplitude and mainly localized near the boundaries of the domain. At this amplitude $(\varepsilon$ $=4.05 \mathrm{~cm} / \mathrm{s}$ ) they are predominantly subharmonic in time, as seen from the two snapshots separated by one forcing period. The average surface does not differ substantially from that predicted by the vibroequilibria theory, being only slightly more deformed (higher at the contact points and lower in the center).

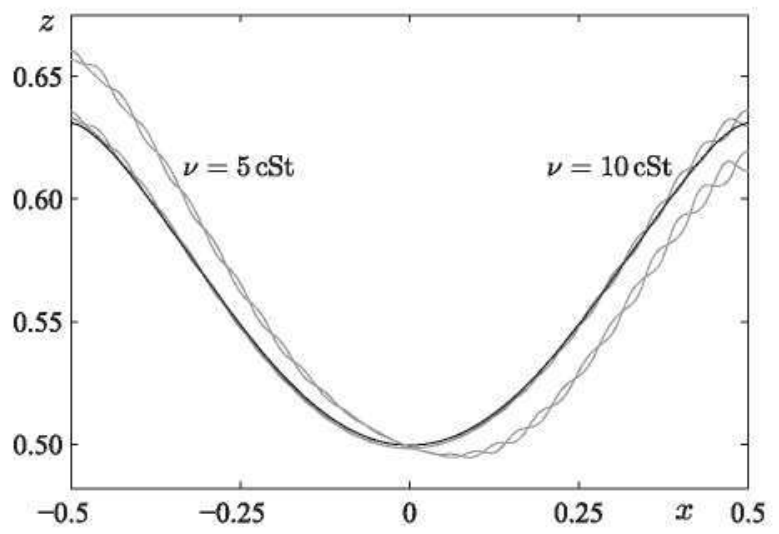

FIG. 18. Comparison of predicted vibroequilibria surface (solid black curve) with surfaces obtained by direct numerical simulation (grey curves) using viscosities of $5 \mathrm{cSt}$ and $10 \mathrm{cSt}$. Two surfaces are shown from each simulation, obtained after $67 \mathrm{~s}$ and separated in time by one forcing period $(0.02 \mathrm{~s})$. Remaining parameters are $V=45 \mathrm{~cm}^{2}, \beta=0^{\circ}, L=9 \mathrm{~cm}, \rho=1 \mathrm{~g} / \mathrm{cm}^{2}$, $\Gamma=20$ dyn, $\varepsilon=4.05 \mathrm{~cm} / \mathrm{s}$, and $\omega / 2 \pi=50 \mathrm{~Hz}$, with the corresponding dimensionless parameters $\nu=5 / 9, \eta=1.85$, and $\mathrm{Bo}=0$.
With viscosity $v=5 \mathrm{cSt}$, the surface waves at the same forcing amplitude are larger and penetrate further into the interior of the domain. At the same time, there is a clear difference in the averaged surface, which is noticeably asymmetric. We show below that this can be attributed mainly to the excitation of an odd sloshing mode that seems to be sustained by coupling to the subharmonic surface waves.

Results presented in this section suggest that the quasistatic surfaces predicted by vibroequilibria theory can be destabilized by sloshing modes. However, sloshing itself is neither surprising nor incompatible with vibroequilibria theory as long as it is transient. Vibroequilibria theory requires that the final state is quasi-steady ${ }^{26}$ (i.e., steady after averaging over the fast time scale of the periodic forcing). Depending on how the forcing is initiated and on the initial configuration of the surface, various transient dynamics can be observed including sloshing, as illustrated in Fig. 19.

If the forcing is turned on quickly with an initially flat surface, the fluid responds rapidly and tends to "overshoot" the vibroequilibria solution. Relaxation toward the final state can take the form of damped low-frequency oscillations (sloshing). On the other hand, if the forcing is applied gradually enough, the fluid may adiabatically follow an "instantaneous" vibroequilibria state, reducing the excitation of transient modes. The manner in which we start the simulations here, from an initially flat surface, benefits from the use of an exponential prefactor $\left[1-\exp \left(-t / T_{0}\right)\right]$ to avoid abrupt motion and numerical instability. This type of initialization naturally excites an even sloshing mode (symmetric about the midplane $x=0$ ) if $T_{0}$ is smaller than its decay time.

A key finding of this section is that surface waves, and particularly subharmonic surface waves, can destabilize the underlying vibroequilibria state by driving a type of sloshing motion that is sustained rather than transient. If this happens, the system no longer exhibits a vibroequilibria solution since

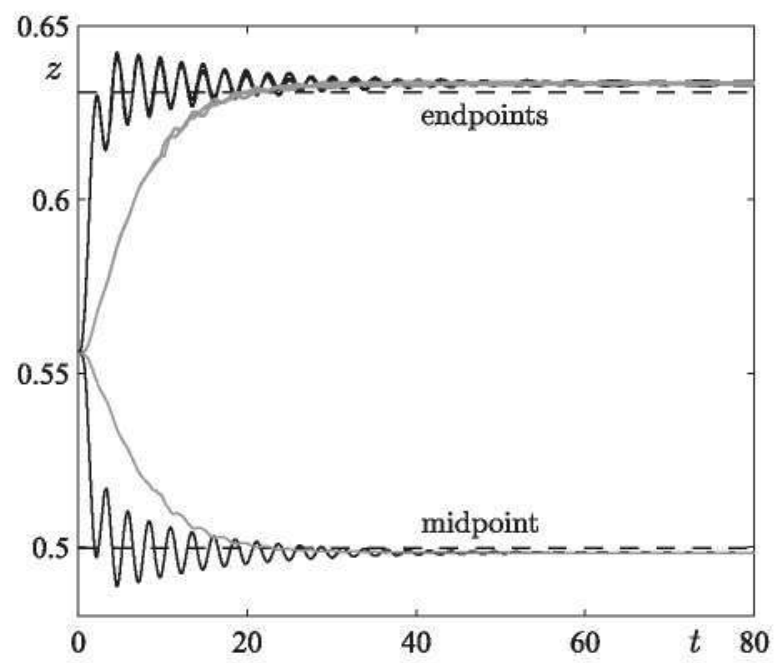

FIG. 19. Filtered height of the two endpoints and the midpoint over time $t$ (in s), after the forcing is turned on with an initially flat surface. Two simulations are shown, both with a prefactor $\left[1-\exp \left(-t / T_{0}\right)\right]$ to smooth the transition to the vibroequilibria. $T_{0}=1$ in the more oscillatory case (solid black curves) and $T_{0}=5$ in the other (solid grey curves). The heights predicted by vibroequilibria theory are shown as dashed lines. Parameters are as in Fig. 18 with $v=10 \mathrm{cSt}$. Most of the rapid harmonic and subharmonic oscillations have been filtered out. 
averaging over the fast time scale of the forcing leaves a modulated time-dependent state. While it may be possible to average again over the longer time scale of the sloshing and recover a quasi-steady surface, this is not the usual meaning of "vibroequilibria" and we will not refer to these slowly oscillating surfaces as such.

The fact that sloshing modes may be easily excited is not surprising in light of Fig. 12. The profile of the total energy $E$ (the sum of vibrational and surface energies) is often relatively flat (when considering variations in surface amplitude) near the vibroequilibria state. This is especially true as one approaches the saddle-node instability where the second derivative of $E$ also vanishes. A slow drift among surfaces having nearly the same energy but different amplitude is possible but, due to viscosity, such amplitude modulations will normally decay over time, as in Fig. 19.

The results presented below suggest that high frequency surface waves can drive these low frequency sloshing modes and support them despite damping. Such persistent sloshing can sometimes be observed when the subharmonic surface waves are large enough to penetrate into the interior of the domain. It is known that (for a nearly flat surface) this situation leads to temporal modulation of the subharmonic waves ${ }^{18,33-35}$ and that this modulation frequency can be very small if the coupling between surface waves emanating from opposite endwalls is weak. We speculate that these modulated subharmonic surface wave states, which introduce a slow frequency to the surface wave dynamics, are the key to effective coupling between the high-frequency surface waves and the lowfrequency sloshing modes that can destabilize vibroequilibria states.

Figure 20 shows the endpoints and midpoint height for a simulation with $5 \mathrm{cSt}$ fluid and $\beta=15^{\circ}$ at a fixed forcing amplitude of $\varepsilon=3.25 \mathrm{~cm} / \mathrm{s}$. Here, most of the rapid harmonic and subharmonic waves are filtered out in order to emphasize the sloshing motion. A spectrogram of the full time series of the right contact point is shown below this to illustrate the frequency content of, in particular, the (modulated) subharmonic waves. After an initial transient as the forcing is switched on with $T_{0}=5$, the subharmonic waves appear at $25 \mathrm{~Hz}$ and are modulated with a frequency of about $1.5 \mathrm{~Hz}$. As the even sloshing mode decays, an odd sloshing mode with frequency of about $0.1 \mathrm{~Hz}$ grows in amplitude, reaching an apparently stable amplitude of approximately 0.01 (peak-to-peak amplitude at the endpoints).

Figure 21 shows the results of three more simulations like that of Fig. 20 but with increasing amplitudes of $\varepsilon=3.5 \mathrm{~cm} / \mathrm{s}$, $3.75 \mathrm{~cm} / \mathrm{s}$, and $4 \mathrm{~cm} / \mathrm{s}$. Again, most of the harmonic and subharmonic content is filtered out of the time series for the endpoints to emphasize the sloshing motion. In each of these simulations, the subharmonic waves are modulated and in each simulation, after an initial transient and the decay of the even sloshing mode, the odd sloshing mode grows. In the final case with $\varepsilon=4 \mathrm{~cm} / \mathrm{s}$ [Fig. 21(c)], this growth does not saturate and the numerical simulation eventually fails. Thus, with sufficiently large forcing, we observe the complete destruction of the vibroequilibria state by the odd sloshing mode (i.e., not even a modulated vibroequilibria remains).

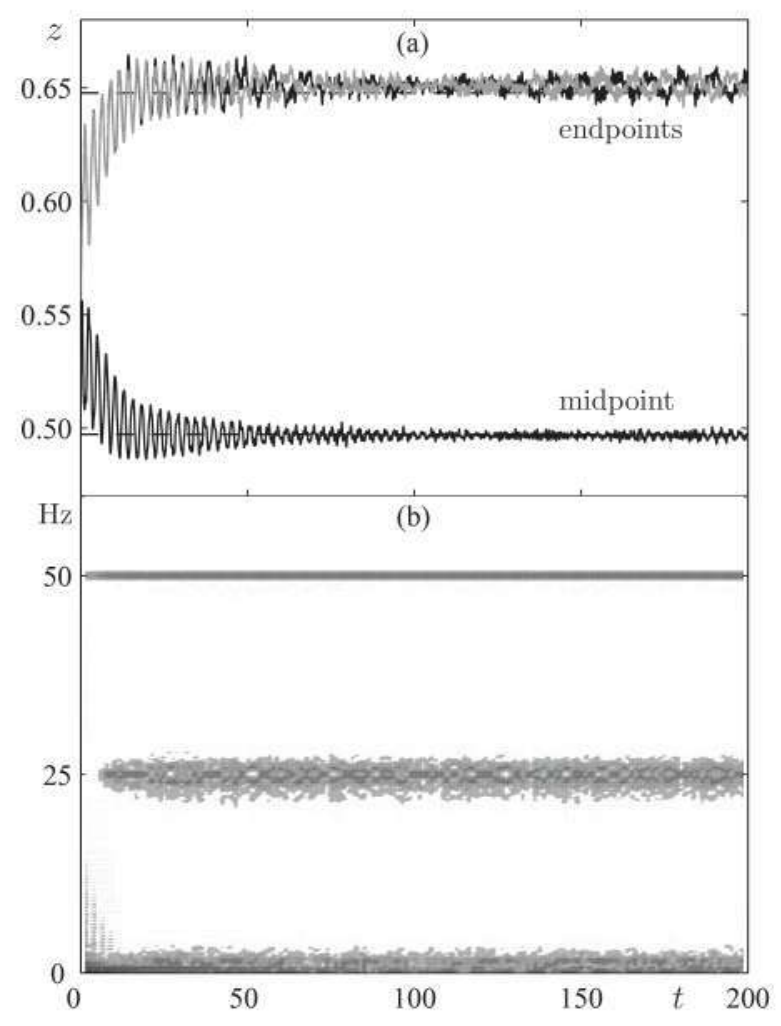

FIG. 20. (a) Filtered height of the two endpoints (left in black, right in gray) and the midpoint over time $t$ (in s), after forcing of amplitude $\varepsilon=3.25 \mathrm{~cm} / \mathrm{s}$, is turned on using the prefactor $[1-\exp (-t / 5)]$. Dashed lines show the prediction of vibroequilibria calculations. (b) Spectrogram of right endpoint showing the presence of harmonic waves at $50 \mathrm{~Hz}$ and modulated subharmonic waves centered at $25 \mathrm{~Hz}$; sloshing frequencies cannot be distinguished at this scale in the transform. Remaining parameters are as in Fig. 18 with $\beta=15^{\circ}$ and $v=5 \mathrm{cSt}$.

The simulations presented above (as well as others that were not included) reveal a complex interaction between transient motion, surface waves, and sloshing motion. For low viscosity fluids, where surfaces waves are more easily excited, the final state is varied and depends on forcing parameters, contact angle, and the initial (transient) approach to the (modulated) vibroequilibria state. At sufficient amplitude both harmonic and subharmonic waves will be present, but it is the subharmonic waves that seem to influence the underlying vibroequilibria state the most, particularly when these waves are modulated (at typical frequencies of 1-3 Hz). Both odd and even sloshing modes may be present. For some parameters, the surface appears to be rendered completely unstable by the growth of the odd sloshing motion.

The most important relationship suggested by the dynamics found in these numerical simulations is the correlation between the growth of the odd sloshing mode and modulated subharmonic surface waves. If modulated subharmonic waves are present, they drive the growth of the odd sloshing mode; this growth may or may not saturate, depending on parameters. The correlation of these two dynamic modes is further demonstrated in Fig. 22, which shows the results of a simulation made by linearly increasing $\varepsilon$ at a rate of $0.0156 \mathrm{~cm} / \mathrm{s}^{2}$ (except for a short initial period of several seconds).

Figure 22(b) shows that the subharmonic waves appear at $\varepsilon \simeq 2 \mathrm{~cm} / \mathrm{s}$, and are modulated initially (until $\varepsilon \simeq 2.18 \mathrm{~cm} / \mathrm{s}$ ). The onset of modulated subharmonic waves coincides with the 


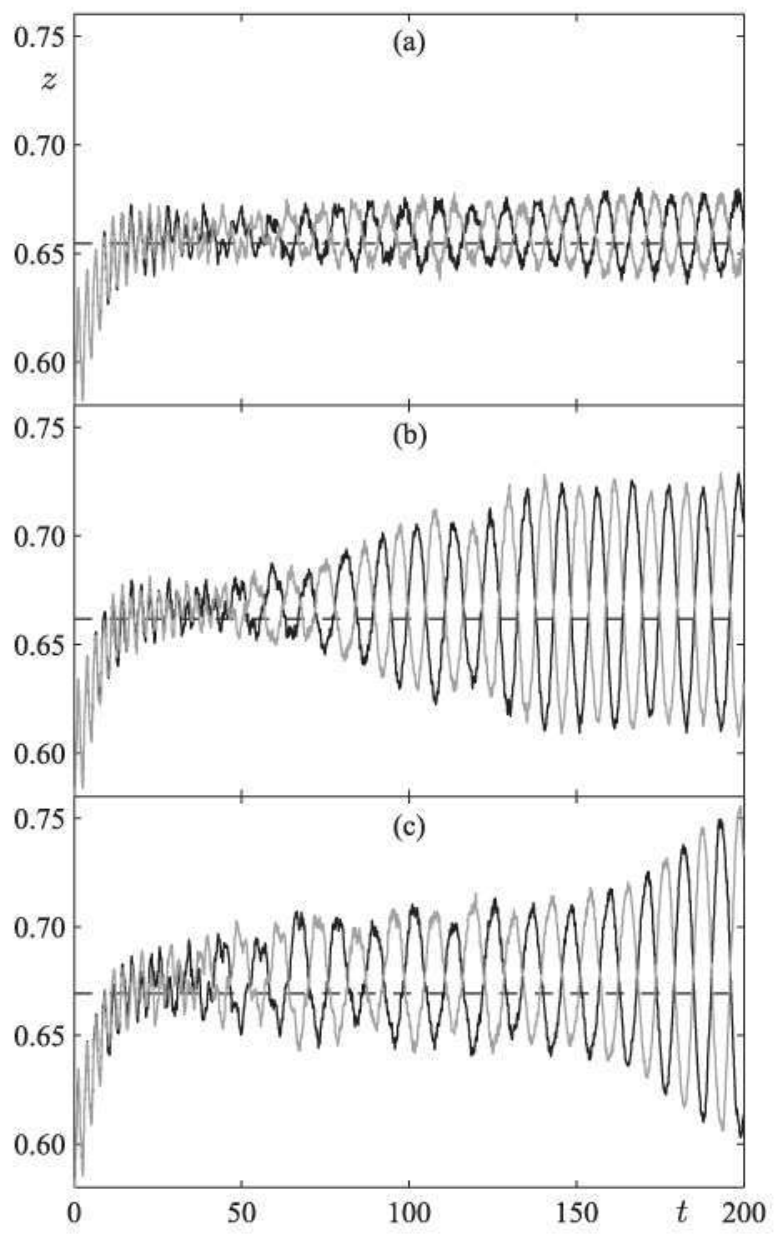

FIG. 21. Filtered height of the two endpoints (left in black, right in gray) over time $t$ (in s) with forcing turned on at $t=0$ using the prefactor $[1-\exp (-t / 5)]$. Dashed lines show the predictions of vibroequilibria calculations. Parameters are as in Fig. 18 with $\beta=15^{\circ}, v=5 \mathrm{cSt}$ and (a) $\varepsilon=3.5 \mathrm{~cm} / \mathrm{s}$, (b) $\varepsilon=$ $3.75 \mathrm{~cm} / \mathrm{s}$, (c) $\varepsilon=4 \mathrm{~cm} / \mathrm{s}$. In each simulation the subharmonic waves are modulated.

rapid growth of the odd sloshing mode. As the (subharmonic) modulation disappears, this odd sloshing motion appears to (slowly) decay. At $\varepsilon \simeq 3.28 \mathrm{~cm} / \mathrm{s}$ the subharmonic modulation returns more vigorously, and with a broader bandwidth than before. This is accompanied by the renewed growth of the odd sloshing mode, which reaches a large enough amplitude to destroy the vibroequilibria state.

We remark that, although the transitions seen in Fig. 22 are likely delayed due to the drift in forcing amplitude, ${ }^{41,42}$ the observation that initial subharmonic modulations disappear with increasing forcing (before returning again at still higher amplitude) is consistent with the scenario presented in the work of Salgado Sánchez et al. ${ }^{35}$ for a horizontally vibrated fluid with no vibroequilibria effect, where the modulated subharmonic surface waves are destroyed in a saddle-node heteroclinic bifurcation and replaced by unmodulated subharmonic surface waves. The current case, complicated both by the vibroequilibria effect and by susceptibility to sloshing modes, is more varied and complex - primary evidence being the reappearance of (irregular) modulations at $\varepsilon \simeq 3.28 \mathrm{~cm} / \mathrm{s}$ in Fig. 22 - but may still reflect several of the key transitions found for a simple pair of coupled parametrically forced subharmonic modes. ${ }^{35}$

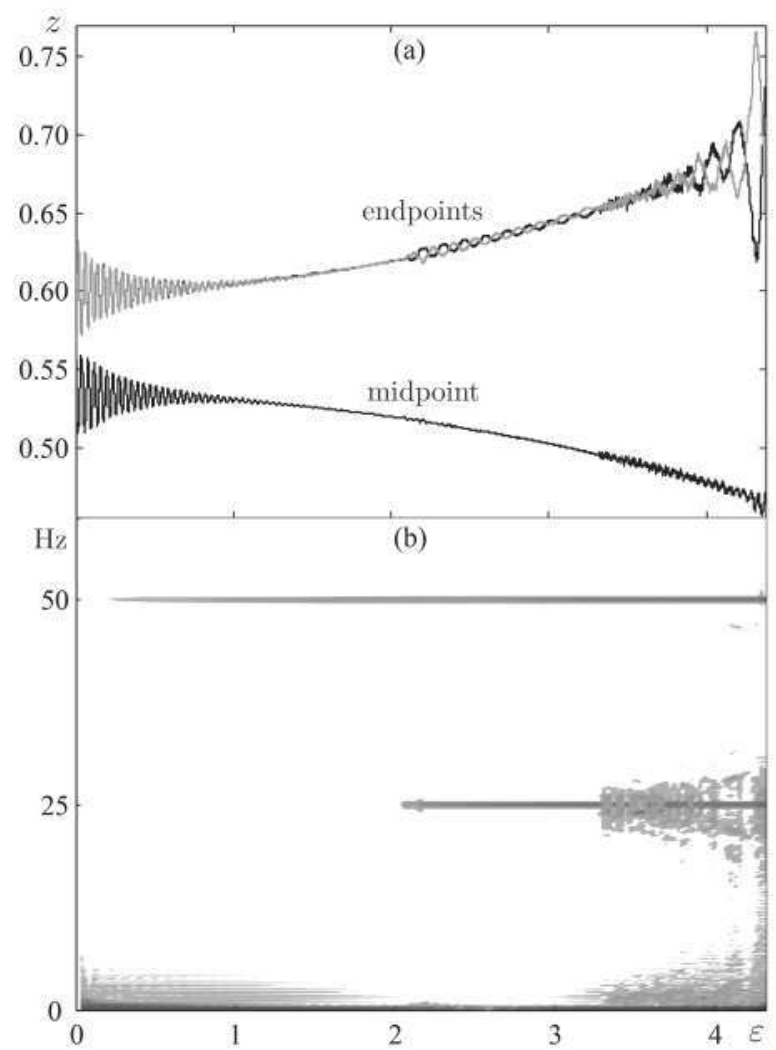

FIG. 22. (a) Filtered height of the two endpoints (left in black, right in gray) and the midpoint as a function of amplitude $\varepsilon$, increasing linearly at a rate of $0.0156 \mathrm{~cm} / \mathrm{s}^{2}$ except for the prefactor $[1-\exp (-t)]$. (b) Spectrogram of right endpoint showing the presence of harmonic waves $(50 \mathrm{~Hz})$ and subharmonic waves $(25 \mathrm{~Hz})$, which are initially modulated, then monochromatic, then modulated more strongly beyond $\varepsilon \simeq 3.2 \mathrm{~cm} / \mathrm{s}$; sloshing frequencies cannot be distinguished at this scale in the transform. Remaining parameters are as in Fig. 18 with $\beta=15^{\circ}$ and $v=5 \mathrm{cSt}$.

\section{CONCLUSIONS}

In this paper, we have examined the predictions of vibroequilibria theory by comparing them with the results of direct numerical simulations of the Navier-Stokes equations. We reviewed selected hydrostatic results in Section III, emphasizing the multiple branches of solutions available and, in particular, the transition as volume is decreased from a unique symmetric connected solution to one of an infinite number of disconnected, generally asymmetric, solutions. It was argued that an analogous transition occurs with vibroequilibria solutions in horizontally vibrated rectangular containers as the forcing, which acts to separate the fluid by pushing it out of the center of the container and toward the two vibrating endwalls, is increased. In many configurations, variations in forcing amplitude will drive hysteretic transitions among distinct vibroequilibria solutions.

The results of combined vibroequilibria calculations and numerical simulations presented in Section V established two main points. First, the theory of vibroequilibria, based on an averaged variational principle, succeeds in describing the average fluid surface over a wide range of forcing values. Second, beyond a minimum (dimensionless) volume $\mathcal{V} \sim 0.36$ of fluid, the symmetric vibroequilibria solution is destroyed not by gradual contact with the container bottom (i.e., contact presaged by gradual downward movement of the midpoint), as it 
is for smaller volumes, but by an instability that occurs prior to this. After this instability the (central dip in the) fluid plunges rapidly toward the bottom, initiating a more violent and unpredictable transition to one of the disconnected vibroequilibria solutions.

This instability was examined in detail in Section VI after restricting the vibroequilibria problem to relevant oneparameter families of surfaces. This simplification allows unstable solutions to be followed and for simple energy profiles to be drawn, revealing the instability as a saddle-node bifurcation where the stable vibroequilibria state collides with an unstable vibroequilibria state of larger amplitude (greater separation along the vibrating endwalls). This instability does not occur in the hydrostatic case, but does in horizontally vibrated rectangular containers of fluid, even when gravity is included. Compelling evidence that this saddle-node instability occurs in the full problem, not just when restricted to particular surface families, was provided by comparing predictions for the dependence of the minimum depth $\delta$ (at the center of the symmetric connected solution) as a function of forcing $\eta$ and volume $\mathcal{V}$.

Hysteresis due to sloshing and other nonlinear effects is expected to be greatly enhanced in sufficiently large fluid volumes where this saddle-node instability occurs. The transition to a disconnected vibroequilibria solution branch is further complicated by dynamic effects related to surface waves and sloshing modes, which were considered in Section VII. Such effects are most relevant for low viscosity fluids, like water, and when surface waves are exited across the interior of the surface, not just in narrow regions near the endwalls. Simulations show that the final state varies with forcing parameters and boundary conditions, and can also depend on the initial (transient) approach to the vibroequilibria state. The final fluid configuration may resemble the vibroequilibria surface predicted by theory, but with harmonic and subharmonic surface waves superimposed on it, or it may deviate from this prediction (see Fig. 18) due to excitation of the first odd and even sloshing modes, which are at much lower frequency than the vibrational forcing.

The most salient conclusion of Section VII is that the interaction between vibroequilibria (i.e., the average surface) and high-frequency surface waves is significantly enhanced by the presence of modulated subharmonic waves. These quasiperiodic solutions, which are typical as well in (nearly) flat horizontally forced systems, ${ }^{18,33-35}$ appear to be crucial in driving the odd sloshing mode because of their slow modulation frequency. If modulated subharmonic waves persist long enough, they can drive the odd sloshing mode to such amplitude that the (modulated) vibroequilibria solution is completely destroyed. We may suppose that the subsequent transition process, which, because of the odd sloshing, is likely to begin from very asymmetric conditions, will lead to increased variation and asymmetry in the final state and, perhaps, for certain parameters, even to repeated cycles of instability, separation/splashing, coalescing, instability, and so on. It will be an interesting direction for further work to examine the interaction between surface waves and average vibroequilibria more carefully, including a more realistic treatment of contact line dynamics and the extension of the vibroequilibria calculations and numerical simulations to the three-dimensional case.

\section{ACKNOWLEDGMENTS}

This work was supported by the Ministerio de Economía y Competitividad under Projects Nos. ESP2013-45432-P and ESP2015-70458-P.

${ }^{1}$ P. M. Gresho and R. L. Sani, "The effects of gravity modulation on the stability of a heated fluid layer," J. Fluid Mech. 40, 783-806 (1970).

${ }^{2} \mathrm{G}$. H. Wolf, "The dynamic stabilization of the Rayleigh-Taylor instability and the corresponding dynamic equilibrium," Z. Phys. 227, 291-300 (1969).

${ }^{3} \mathrm{G}$. H. Wolf, "Dynamic stabilization of the interchange instability of a liquidgas interface," Phys. Rev. Lett. 24, 444-446 (1970).

${ }^{4}$ J. M. Vega, E. Knobloch, and C. Martel, "Nearly inviscid Faraday waves in annular containers of moderately large aspect ratio," Physica D 154, 313-336 (2001).

${ }^{5}$ M. Faraday, "On a peculiar class of acoustical figures; and on certain forms assumed by groups of particles upon vibrating elastic surfaces," Philos. Trans. R. Soc. London 121, 299-340 (1831).

${ }^{6} \mathrm{~J}$. Miles and D. Henderson, "Parametrically forced surface waves," Annu. Rev. Fluid Mech. 22, 143-165 (1990).

${ }^{7}$ T. B. Benjamin and F. Ursell, "The stability of a plane free surface of a liquid in vertical periodic motion," Proc. R. Soc. A 225, 505-515 (1954).

${ }^{8}$ W. S. Edwards and S. Fauve, "Patterns and quasi-patterns in the Faraday experiment," J. Fluid Mech. 278, 123-148 (1994).

${ }^{9}$ A. Kudrolli, B. Pier, and J. P. Gollub, "Superlattice patterns in surface waves," Physica D 123, 99-111 (1998).

${ }^{10} \mathrm{H}$. Arbell and J. Fineberg, "Pattern formation in two-frequency forced parametric waves," Phys. Rev. E 65, 036224 (2002).

${ }^{11} \mathrm{~J}$. W. Miles, "Resonantly forced surface waves in a circular container," J. Fluid Mech. 149, 15-31 (1984).

${ }^{12} \mathrm{M}$. Funakoshi and S. Inoue, "Surface waves due to resonant horizontal oscillation," J. Fluid Mech. 192, 219-247 (1988).

${ }^{13} \mathrm{Z}$. C. Feng, "Transition to traveling waves from standing waves in a rectangular container subjected to horizontal excitations," Phys. Rev. Lett. 79, 415-418 (1997).

${ }^{14}$ B. J. S. Barnard and W. G. Pritchard, "Cross-waves. Part 2. Experiments," J. Fluid Mech. 55, 245-255 (1972).

${ }^{15}$ C. J. R. Garrett, "On cross-waves," J. Fluid Mech. 41, 837-849 (1970).

${ }^{16}$ A. F. Jones, "The generation of cross-waves in a long deep channel by parametric resonance,” J. Fluid Mech. 138, 53-74 (1984).

${ }^{17} \mathrm{~F}$. Varas and J. M. Vega, "Modulated surface waves in large-aspect-ratio horizontally vibrated containers," J. Fluid Mech. 579, 271-304 (2007).

${ }^{18}$ I. Tinao, J. Porter, A. Laverón-Simavilla, and J. Fernández, "Cross-waves excited by distributed forcing in the gravity-capillary regime," Phys. Fluids 26, 024111 (2014).

${ }^{19}$ D. V. Lyubimov and A. A. Cherepanov, "Development of a steady relief at the interface of fluids in a vibrational field," Fluid Dyn. 21, 849-854 (1986) [Izvestiya Akademii Nauk SSSR, Mekhanika Zhidkosti i Gaza (in Russian)].

${ }^{20} \mathrm{E}$. Talib, S. V. Jalikop, and A. Juel, "The influence of viscosity on the frozen wave instability: Theory and experiment,"J. Fluid Mech. 584, 45-68 (2007).

${ }^{21}$ Y. A. Gaponenko, M. Torregrosa, V. Yasnou, A. Mialdun, and V. Shevtsova, "Dynamics of the interface between miscible liquids subjected to horizontal vibration," J. Fluid Mech. 784, 342-372 (2015).

${ }^{22}$ S. V. Jalikop and A. Juel, "Steep capillary-gravity waves in oscillatory sheardriven flows," J. Fluid Mech. 640, 131-150 (2009).

${ }^{23} \mathrm{~S}$. V. Jalikop and A. Juel, "Oscillatory transverse instability of interfacial waves in horizontally oscillating flows," Phys. Fluids 24, 044104 (2012).

${ }^{24}$ M. V. Khenner, D. V. Lyubimov, T. S. Belozerova, and B. Roux, "Stability of plane-parallel vibrational flow in a two-layer system," Eur. J. Mech. B-Fluid 18, 1085-1101 (1999).

${ }^{25}$ G. Gandikota, D. Chatain, S. Amiroudine, T. Lyubimova, and D. Beysens, "Frozen-wave instability in near-critical hydrogen subjected to horizontal vibration under various gravity fields," Phys. Rev. E 89, 012309 (2014).

${ }^{26}$ D. V. Lyubimov, A. A. Cherepanov, T. P. Lyubimova, and B. Roux, "Interface orienting by vibration," C. R. Acad. Sci., Ser. Ilb: Mec., Phys., Chim., Astron. 325, 391-396 (1997).

${ }^{27}$ K. Beyer, I. Gawriljuk, M. Günther, I. Lukovsky, and A. Timokha, "Compressible potential flows with free boundaries. Part I: Vibrocapillary equilibria," Z. Angew. Math. Mech. 81, 261-271 (2001).

${ }^{28} \mathrm{~K}$. Beyer, M. Günther, and A. Timokha, "Variational and finite element analysis of vibroequilibria," Comput. Methods Appl. Math. 4, 290-323 (2004). 
${ }^{29}$ I. Gavrilyuk, I. Lukovsky, and A. Timokha, "Two-dimensional variational vibroequilibria and Faraday's drops," Z. Angew. Math. Phys. 55, 1015-1033 (2004).

${ }^{30}$ P. L. Kapitza, "Pendulum with a vibrating suspension," Usp. Fiz. Nauk 44, $7-15$ (1951).

${ }^{31}$ R. F. Ganiev, V. D. Lakiza, and A. S. Tsapenko, "Dynamic behavior of the free liquid surface subject to vibrations under conditions of near-zero gravity," Sov. Appl. Mech. 13, 499-503 (1977) [Prikladnaya Mekhanika (in Russian)].

${ }^{32}$ D. V. Lyubimov, T. P. Lyubimova, and A. A. Cherepanov, Dynamics of Interfaces in Vibrational Fields (Fizmatlit, 2003) (in Russian).

${ }^{33}$ J. Porter, I. Tinao, A. Laverón-Simavilla, and J. Rodríguez, "Onset patterns in a simple model of localized parametric forcing," Phys. Rev. E 88, 042913 (2013).

${ }^{34}$ J. M. Perez-Gracia, J. Porter, F. Varas, and J. M. Vega, "Subharmonic capillary-gravity waves in large containers subject to horizontal vibrations," J. Fluid Mech. 739, 196-228 (2014).
${ }^{35}$ P. Salgado Sánchez, J. Porter, I. Tinao, and A. Laverón-Simavilla, "Dynamics of weakly coupled parametrically forced oscillators," Phys. Rev. E 94, 022216 (2016).

${ }^{36}$ G. B. Whitman, Linear and Nonlinear Waves (John Wiley \& Sons, 1974).

${ }^{37}$ J. A. Nicolás and J. M. Vega, "Three-dimensional streaming flows driven by oscillatory boundary layers," Fluid Dyn. Res. 32, 119-139 (2003).

${ }^{38}$ MATLAB 2013a, The MathWorks, Inc., Natick, Massachusetts, USA.

${ }^{39}$ See https://www.comsol.com for COMSOL Multiphysics 3.5a.

${ }^{40}$ J. Porter, I. Tinao, A. Laverón-Simavilla, and C. A. Lopez, "Pattern selection in a horizontally vibrated container," Fluid Dyn. Res. 44, 065501 (2012).

${ }^{41}$ P. Mandel and T. Erneux, "The slow passage through a steady bifurcation: Delay and memory effects," J. Stat. Phys. 48, 1059-1070 (1987).

${ }^{42}$ S. M. Baer, T. Erneux, and J. Rinzel, "'The slow passage through a Hopf bifurcation: Delay, memory effects, and resonance," SIAM J. Appl. Math. 49, 55-71 (1989). 